DOI 10.4171/JEMS/264

Lucian Beznea

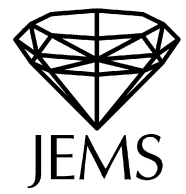

\title{
Potential-theoretical methods in the construction of measure-valued Markov branching processes
}

Received October 5, 2008 and in revised form February 13, 2009

\begin{abstract}
We develop potential-theoretical methods in the construction of measure-valued branching processes. We complete results of P. J. Fitzsimmons and E. B. Dynkin on the construction, regularity and other properties of the superprocess associated with a given right process and a branching mechanism.
\end{abstract}

Keywords. Branching process, superprocess, excessive function, right process

\section{Introduction}

Let $X=\left(\Omega, \mathcal{F}, \mathcal{F}_{t}, \theta_{t}, X_{t}, P^{x}\right)$ be a fixed Borel right Markov process with state space $E$, a Lusin topological space with Borel $\sigma$-algebra $\mathcal{B}=\mathcal{B}(E)$. Let $\left(P_{t}\right)_{t \geq 0}$ be the transition semigroup of $X, P_{t} f(x)=E^{x}\left(f \circ X_{t} ; t<\zeta\right)$, and $\left(U_{\alpha}\right)_{\alpha>0}$ the associated resolvent of kernels, $U_{\alpha} f=\int_{0}^{\infty} e^{-\alpha t} P_{t} f d t$ for all $f \in \mathrm{p} \mathcal{B} ; \mathrm{p} \mathcal{B}$ denotes the set of all positive numerical $\mathcal{B}$-measurable functions on $E$.

We also fix a "branching mechanism", that is, a function $\Phi: E \times[0, \infty) \rightarrow \mathbb{R}$ of the form

$$
\Phi(x, \lambda)=-b(x) \lambda-c(x) \lambda^{2}+\int_{0}^{\infty}\left(1-e^{-\lambda s}-\lambda s\right) N(x, d s)
$$

where $c \geq 0$ and $b$ are bounded $\mathcal{B}$-measurable functions and $N: \mathrm{p} \mathcal{B}((0, \infty)) \rightarrow \mathrm{p} \mathcal{B}(E)$ is a kernel such that $N\left(u \wedge u^{2}\right) \in \mathrm{bp} \mathcal{B}$ and $\lim _{\eta \searrow 0}\left\|N\left(1_{(0, \eta]} \cdot u^{2}\right)\right\|_{\infty}=0$. For a family $\mathcal{F}$ of numerical valued functions (defined on the same given set) we denote by $b \mathcal{F}$ those elements of $\mathcal{F}$ that are bounded.

Notice that examples of branching mechanisms are $\Phi(\lambda)=-\lambda^{\beta}$ for $1<\beta \leq 2$ since

$$
\lambda^{\alpha}=\frac{\alpha}{\Gamma(1-\alpha)} \int_{0}^{\infty} \frac{1-e^{-\lambda s}}{s^{\alpha+1}} d s
$$

and therefore

$$
-\lambda^{\alpha+1}=\frac{\alpha(\alpha+1)}{\Gamma(1-\alpha)} \int_{0}^{\infty} \frac{1-e^{-\lambda s}-\lambda s}{s^{\alpha+2}} d s \quad \text { if } 0<\alpha<1 .
$$

L. Beznea: "Simion Stoilow" Institute of Mathematics of the Romanian Academy, P.O. Box 1-764, RO-014700 Bucureşti, Romania; e-mail: lucian.beznea@imar.ro

Mathematics Subject Classification (2010): Primary 60J45, 60J80; Secondary 60J40, 60J35, 47D07 
In this paper we develop potential-theoretical methods related to the construction of an $(X, \Phi)$-superprocess. The method of construction is the so called "semigroup approach" presented in Ch. 4 of the book [11] of E. B. Dynkin; cf. also the references therein. P. J. Fitzsimmons [13] obtained with this approach regularity properties of the superprocess (which is in particular a right process); see also the papers [10] and [15] for extensions and improvements, including the inhomogeneous case. The property of an $(X, \Phi)$-superprocess to be a right process is essential for further developments like considering the capacity induced by the reduction operator (the capacity of the superprocess), the stochastic calculus with additive and multiplicative functionals of the superprocess, and the Revuz correspondence (see e.g. [2] and [17] for the general theory).

We shall emphasize (in Corollary 4.3 relations between the excessive functions with respect to $X$ and two classes of excessive functions (defined on $M(E):=$ the space of all positive finite measures on $(E, \mathcal{B})$ ), with respect to the (forthcoming) $(X, \Phi)$ superprocess: the "exponential type" excessive functions which will be useful in the construction and in proving the properties of the transition semigroup of the superprocess, and the "linear" ones which will be used to obtain relations between the reduction operators of $X$ and, respectively, the $(X, \Phi)$-superprocess (cf. Proposition 4.7). A special linear excessive function for the superprocess becomes a function having compact level sets which in particular will provide a nest of compacts on $M(E)$.

The excessive measures (which correspond to the excessive functions for the dual theory), in particular the potential excessive measures of an $(X, \Phi)$-superprocess, as well as the energy functional (a bilinear functional between excessive functions and measures generalizing the classical "mutual energy") will also be studied. Based on these tools we shall give (in Theorem 4.9 the proof of the existence of an $(X, \Phi)$-superprocess, a Borel right process with state space $M(E)$ endowed with the weak topology. Our proof is transparent, the main arguments coming from the potential theory associated with Markov processes. In addition, it turns out that the entrance space of an $h$-transform of the $(X, \Phi)$ superprocess is precisely $M\left(E_{1}\right)$, where $E_{1}$ is the entrance space of $X$. This result should be compared with the similar ones obtained by P. J. Fitzsimmons [13, Theorem (3.7)] and by E. B. Dynkin [9]. The existence of a nest of weak compact sets on the space of measures will ensure that the superprocess has càdlàg trajectories.

We complete the introduction with the outline of the construction of the measurevalued branching Markov process associated with $X$ and $\Phi$, the $(X, \Phi)$-superprocess. It will be done in three steps. The first two steps follow the approach from [13].

I. The construction of a non-linear semigroup. For each $f \in \operatorname{bp} \mathcal{B}$ the equation

$$
v_{t}(x)=P_{t} f(x)+\int_{0}^{t} P_{s}\left(x, \Phi\left(\cdot, v_{t-s}\right)\right) d s, \quad t \geq 0, x \in E,
$$

has a unique solution $(t, x) \mapsto V_{t} f(x)$ jointly measurable in $(t, x)$ and such that $\sup _{0 \leq s \leq t}\left\|v_{s}\right\|_{\infty}<\infty$ for all $t>0$. The mappings $f \mapsto V_{t} f$ form a nonlinear semigroup of operators on $\mathrm{bp} \mathcal{B}$.

Precise results on the nonlinear semigroup will be given in Section 3 (Proposition 3.2); we are indebted to P. J. Fitzsimmons for providing us with a manuscript containing the 
proof of Proposition 2.3 of [13]. The proofs of the results from Section 3 will be presented in the Appendix.

II. The transition semigroup on the space of measures. For a function $f \in \mathrm{bp} \mathcal{B}$ we shall consider the mappings $l_{f}: M(E) \rightarrow \mathbb{R}$ and $e_{f}: M(E) \rightarrow[0,1]$ defined by

$$
l_{f}(\mu):=\langle\mu, f\rangle:=\int f d \mu, \quad \mu \in M(E), \quad e_{f}:=\exp \left(-l_{f}\right) .
$$

$M(E)$ is endowed with the $\sigma$-algebra $\mathcal{M}(E)$ generated by $\left\{l_{f} \mid f \in \mathrm{bp} \mathcal{B}\right\}$.

For each $t \geq 0$ there exists a unique kernel $Q_{t}$ on $(M(E), \mathcal{M}(E))$ such that

$$
Q_{t}\left(e_{f}\right)=e_{V_{t} f}, \quad f \in \mathrm{bp} \mathcal{B} .
$$

Since the family $\left(V_{t}\right)_{t \geq 0}$ is a (nonlinear) semigroup on bp $\mathcal{B},\left(Q_{t}\right)_{t \geq 0}$ is a (linear) semigroup of kernels on $(M(E), \mathcal{M}(E))$.

III. The measure-valued Markov process. This step is essentially different from the existing approaches. We first show (Proposition 4.5p that all the points of $M(E)$ are nonbranch points for the semigroup $\left(Q_{t}\right)_{t \geq 0}$, consequently it becomes the transition function of a right Markov process having a larger space state, namely the entrance space $M(E)_{1}$. In order to prove that the process stays in $M(E)$, under some regularity conditions on $\Phi$ or $\left(V_{t}\right)_{t \geq 0}$, we show that the set $M(E)_{1} \backslash M(E)$ is polar (see (2.4) below) and so there exists a Borel right process with state space $M(E)$ endowed with the weak topology, having $\left(Q_{t}\right)_{t \geq 0}$ as transition semigroup; this is precisely the $(X, \Phi)$-superprocess. A key argument in our development is a measure representation for negative definite functions defined on the convex cone of all bounded excessive functions (with respect to $X$ ); cf. Proposition 2.4 It is inspired by a measure-theoretical result of P. J. Fitzsimmons (Corollary (A.6) in [13] ) which will be obtained here as a consequence.

\section{Sub-Markovian resolvents of kernels and negative definite functions}

Below we follow the terminology of [2]. Let $\mathcal{U}=\left(U_{\alpha}\right)_{\alpha>0}$ be a sub-Markovian resolvent of kernels on the Lusin measurable space $(E, \mathcal{B})$. We shall denote by $U$ the initial kernel of $\mathcal{U}: U=\sup _{\alpha>0} U_{\alpha}$. If $\beta>0$ then the family $\mathcal{U}_{\beta}=\left(U_{\beta+\alpha}\right)_{\alpha>0}$ is also a sub-Markovian resolvent of kernels on $(E, \mathcal{B})$, having $U_{\beta}$ as (bounded) initial kernel. A function $v \in \mathrm{p} \mathcal{B}$ is called $\mathcal{U}$-supermedian if $\alpha U_{\alpha} v \leq v$ for all $\alpha>0$. A $\mathcal{U}$-supermedian function $v$ is named $\mathcal{U}$-excessive if in addition $\sup _{\alpha>0} \alpha U_{\alpha} v=v$. We denote by $\mathcal{E}(\mathcal{U})(\operatorname{resp} . \mathcal{S}(\mathcal{U})$ ) the set of all $\mathcal{B}$-measurable $\mathcal{U}$-excessive functions (resp. $\mathcal{B}$-measurable $\mathcal{U}$-supermedian functions). If $v \in \mathcal{S}(\mathcal{U})$ then the function $\widehat{v}:=\sup _{\alpha>0} \alpha U_{\alpha} v$ is $\mathcal{U}$-excessive and the set $M=[v \neq \widehat{v}]$ is $\mathcal{U}$-negligible, i.e., $U_{\beta}\left(1_{M}\right)=0$ for some (and hence all) $\beta>0$. We denote by $D_{\mathcal{U}}$ the set of all non-branch points with respect to $\mathcal{U}$, namely,

$$
\left.D_{\mathcal{U}}:=\{x \in E \mid \inf (v, u)(x)=\widehat{\inf (v, u})(x) \text { for all } v, u \in \mathcal{E}(\mathcal{U}), \widehat{1}(x)=1(x)\right\} .
$$

If $\beta>0$ then $D_{\mathcal{U}_{\beta}} \in \mathcal{B}$ and it does not depend on $\beta>0$, and $E \backslash D_{\mathcal{U}_{\beta}}$ is $\mathcal{U}$-negligible. 
Recall that a $\sigma$-finite measure $\xi$ on $(E, \mathcal{B})$ is called $\mathcal{U}$-excessive if $\xi \circ \alpha U_{\alpha} \leq \xi$ for all $\alpha>0$. We denote by $\operatorname{Exc}(\mathcal{U})$ the set of all $\mathcal{U}$-excessive measures. A $\mathcal{U}$-excessive measure of the form $\mu \circ U$ (where $\mu$ is a $\sigma$-finite measure) is called potential. We denote by $\operatorname{Pot}(\mathcal{U})$ the convex cone of all potential $\mathcal{U}$-excessive measures. Further let $L: \operatorname{Exc}(\mathcal{U}) \times \mathcal{E}(\mathcal{U})$ $\rightarrow \overline{\mathbb{R}}_{+}$be the energy functional (associated with $\mathcal{U}$ ) defined by

$$
L(\xi, v):=\sup \{\mu(v) \mid \operatorname{Pot}(\mathcal{U}) \ni \mu \circ U \leq \xi\}
$$

for all $\xi \in \operatorname{Exc}(\mathcal{U})$ and $v \in \mathcal{E}(\mathcal{U})$. The energy functional associated with $\mathcal{U}_{\beta}$ will be denoted by $L_{\beta}$.

For the rest of the section (with the exception of Corollary 2.3) we assume that for some (and hence all) $\beta>0$ :

- The pointwise infimum of any two $\mathcal{U}_{\beta}$-excessive functions is also a $\mathcal{U}_{\beta}$-excessive function and $1 \in \mathcal{E}(\mathcal{U})$ (or, equivalently, $D_{\mathcal{U}_{\beta}}=E$ ).

- $\sigma\left(\mathcal{E}\left(\mathcal{U}_{\beta}\right)\right)=\mathcal{B}$.

Notice that if $\mathcal{U}$ is the resolvent of a Borel right process with state space $E$, then the two conditions above are satisfied.

The fine topology on $E$ is the topology generated by all $\mathcal{U}_{\beta}$-excessive functions (and it does not depend on $\beta>0$ ).

A metrizable topology on $E$ is called natural if it is smaller than the fine topology and its Borel $\sigma$-algebra is $\mathcal{B}$. Every Ray topology (i.e., the topology generated by a Ray cone; see e.g. [2] for details) as well as the original topology on $E$ are natural.

We now present several results related to the existence of a right process having $\mathcal{U}$ as associated resolvent.

(2.1) The following assertions are equivalent:

(2.1.a) For some (and hence all) $\beta>0$, in $\operatorname{Exc}\left(\mathcal{U}_{\beta}\right)$ every $\mathcal{U}_{\beta}$-excessive measure dominated by a potential is also a potential.

(2.1.b) There exists a Lusin topology on $E$ such that $\mathcal{B}$ is the $\sigma$-algebra of all Borel sets of $E$, and there exists a right process with state space $E$, having $\mathcal{U}$ as the associated resolvent.

(2.1.c) For every natural topology on $E$ there exists a right process with state space $E$, having $\mathcal{U}$ as associated resolvent.

(See Section 1.7 in [2] and Theorem 1.3 in [3].)

Remark. By Proposition 3.5.3 in [2], for every natural topology there exists on $E$ a finer Ray topology. In particular we deduce from the above considerations that for a right process the original topology may always be enlarged to a Ray one, considering the so called "Ray realization of the process".

(2.2) The following property (satisfied for one and therefore for all $\beta>0$ ) implies that the above equivalent assertions (2.1.a), (2.1.b) and (2.1.c) hold:

(2.2.a) every $\xi \in \operatorname{Exc}\left(\mathcal{U}_{\beta}\right)$ with $L_{\beta}(\xi, 1)<\infty$ is a potential. 
(2.3) There exists a second Lusin measurable space $\left(E_{1}, \mathcal{B}_{1}\right)$ such that $E \subset E_{1}, E \in$ $\mathcal{B}_{1}, \mathcal{B}=\left.\mathcal{B}_{1}\right|_{E}$, and a resolvent of kernels $\mathcal{U}^{1}=\left(U_{\alpha}^{1}\right)_{\alpha>0}$ on $\left(E_{1}, \mathcal{B}_{1}\right)$ such that $D_{\mathcal{U}_{\beta}^{1}}=E_{1}, \sigma\left(\mathcal{E}\left(\mathcal{U}_{\beta}^{1}\right)\right)=\mathcal{B}_{1}, U_{\beta}^{1}\left(1_{E_{1} \backslash E}\right)=0, E_{1}$ satisfies (2.2.a) with respect to $\mathcal{U}^{1}$, and $\mathcal{U}$ is the restriction of $\mathcal{U}^{1}$ to $E$ (i.e. $U_{\beta}(g)=U_{\beta}^{1}\left(g^{1}\right)$, where $g^{1} \in \mathrm{p} \mathcal{B}_{1}$ and $\left.g^{1}\right|_{E}=g$ ). In particular, by (2.1) and (2.2), $\mathcal{U}^{1}$ is the resolvent of a right process with state space $E_{1}$, for a suitable Lusin topology on $E_{1}$. More precisely, one can take for $E_{1}$ the set of all extreme points of the set $\left\{\xi \in \operatorname{Exc}\left(\mathcal{U}_{\beta}\right) \mid L_{\beta}(\xi, 1)=1\right\}$, endowed with the $\sigma$-algebra $\mathcal{B}_{1}$ generated by the functionals $\tilde{u}, \widetilde{u}(\xi):=L_{\beta}(\xi, u)$ for all $\xi \in E_{1}$ and $u \in \mathcal{E}\left(\mathcal{U}_{\beta}\right)$. The set $E_{1}$ is called the saturation of $E$. If $\mathcal{U}$ is the resolvent of a right process with state space $E$, then $E_{1}$ coincides with the entrance space of the process. Let $\left(E^{\prime}, \mathcal{B}^{\prime}\right)$ be a Lusin measurable space such that $E \subset E^{\prime}$, $E \in \mathcal{B}^{\prime}, \mathcal{B}=\left.\mathcal{B}^{\prime}\right|_{E}$, and there exists a proper sub-Markovian resolvent of kernels $\mathcal{U}^{\prime}=\left(U_{\alpha}^{\prime}\right)_{\alpha>0}$ on $\left(E^{\prime}, \mathcal{B}^{\prime}\right)$ with $D_{\mathcal{U}_{\beta}^{\prime}}=E^{\prime}, \sigma\left(\mathcal{E}\left(\mathcal{U}_{\beta}^{\prime}\right)\right)=\mathcal{B}^{\prime}, U_{\beta}^{\prime}\left(1_{E^{\prime} \backslash E}\right)=0, E^{\prime}$ satisfies (2.2.a) with respect to $\mathcal{U}^{\prime}$, and $\mathcal{U}$ is the restriction of $\mathcal{U}^{\prime}$ to $E$. Then the map $x \mapsto \varepsilon_{x} \circ U_{\beta}^{\prime}$ is a measurable isomorphism between $\left(E^{\prime}, \mathcal{B}^{\prime}\right)$ and the measurable space $\left(E_{1}, \mathcal{B}_{1}\right)$.

(2.4) The following property is equivalent to the above assertions (2.1.a), (2.1.b) and (2.1.c): the set $E_{1} \backslash E$ is polar with respect to the right process on $E_{1}$ given by (2.3).

Further in this section, for some fixed $\beta>0$ we shall use the notation

$$
\mathcal{S}=\mathrm{b} \mathcal{E}\left(\mathcal{U}_{\beta}\right)
$$

(2.5) Let $h \in \mathcal{E}(\mathcal{U}), h>0$. We consider the kernels $U_{\alpha}^{h}$ on $(E, \mathcal{B})$ defined by $U_{\alpha}^{h} f:=$ $(1 / h) U_{\alpha}(h f), f \in \mathrm{p} \mathcal{B}$. Then the family $\mathcal{U}^{h}=\left(U_{\alpha}^{h}\right)_{\alpha>0}$ is also a sub-Markovian resolvent of kernels on $(E, \mathcal{B}), D_{\mathcal{U}^{h}}=E$ and the following assertions hold:

(i) $\mathcal{E}\left(\mathcal{U}_{\beta}^{h}\right)=(1 / h) \cdot \mathcal{E}\left(\mathcal{U}_{\beta}\right)$ and if $\xi$ is a $\sigma$-finite measure on $(E, \mathcal{B})$, then

$$
\xi \in \operatorname{Exc}\left(\mathcal{U}_{\beta}\right) \Leftrightarrow h \cdot \xi \in \operatorname{Exc}\left(\mathcal{U}_{\beta}^{h}\right), \quad \xi \in \operatorname{Pot}\left(\mathcal{U}_{\beta}\right) \Leftrightarrow h \cdot \xi \in \operatorname{Pot}\left(\mathcal{U}_{\beta}^{h}\right)
$$

In particular, $E$ satisfies (2.1.a) with respect to $\mathcal{U}$ and $\mathcal{U}^{h}$ simultaneously.

(ii) If $\xi \in \operatorname{Exc}\left(\mathcal{U}_{\beta}\right)$ and $u \in \mathcal{E}\left(\mathcal{U}_{\beta}\right)$, then $L_{\beta}(\xi, u)=L_{\beta}^{h}(h \cdot \xi, u / h)$, where $L_{\beta}^{h}$ denotes the energy functional with respect to $\mathcal{U}_{\beta}^{h}$. The set $E$ satisfies (2.2.a) with respect to $\mathcal{U}^{h}$ if and only if every $\xi \in \operatorname{Exc}\left(\mathcal{U}_{\beta}\right)$ with $L_{\beta}(\xi, h) \leq 1$ is a potential.

Lemma 2.1. Assume that $E$ satisfies (2.2.a) with respect to $\mathcal{U}$ and there exists $k>0$ such that $U_{\beta} 1 \geq k$. Let $\gamma$ be a positive finite measure on a second measurable space $\left(F, \mathcal{B}_{F}\right)$ (not necessarily of Lusin type) and let $K: \mathcal{S} \rightarrow \mathrm{p} \mathcal{B}_{F}$ be a mapping such that: $K 0=0$, $\gamma$-a.e., $K u<\infty$ for all $u \in \mathcal{S}$, and if $\left(u_{n}\right)_{n} \subset \mathcal{S}$ with $u=\sum_{n} u_{n}$, then $K u=\sum_{n} K u_{n}$, $\gamma$-a.e. Then there exists a kernel $\widetilde{K}: \mathrm{bp} \mathcal{B} \rightarrow \mathrm{p} \mathcal{B}_{F}$ such that for all $f \in \mathrm{bp} \mathcal{B}$ we have $\widetilde{K} U_{\beta} f=K U_{\beta} f, \gamma$-a.e. 
Proof. Let us consider the mapping $K^{\prime}: \mathrm{bp} \mathcal{B} \rightarrow \mathrm{p} \mathcal{B}_{F}$ defined by $K^{\prime}=K \circ U_{\beta}$. Then $K^{\prime}$ is a $\gamma$-quasi kernel and, in addition, $K^{\prime} \circ \alpha U_{\beta+\alpha} f \leq K^{\prime} f \gamma$-a.e. for all $\alpha>0$ and $f \in \mathrm{bp} \mathcal{B}$. So, there exists a kernel $\underset{\widetilde{\widetilde{K}}}{\widetilde{\widetilde{K}}} \mathrm{bp} \mathcal{B} \rightarrow \mathrm{p} \mathcal{B}_{F}$ such that $\widetilde{\widetilde{K}} f<\infty$ for all $f \in \mathrm{bp} \mathcal{B}$, $\widetilde{\widetilde{K}} \circ \alpha U_{\beta+\alpha} \leq \widetilde{\widetilde{K}}$ for all $\alpha \in \mathbb{Q}_{+}, \widetilde{\widetilde{K}} 1<\infty$ and $K U_{\beta} f=\widetilde{\widetilde{K}} f \gamma$-a.e. for all $f \in$ bp $\mathcal{B}$ (see e.g. [2]). Consequently, for every $x \in F$ the functional $f \stackrel{\widetilde{K}_{x}}{\longmapsto} \widetilde{\widetilde{K}} f(x)$ is a $\mathcal{U}_{\beta}$-excessive measure on $E$ and $L_{\beta}\left(\widetilde{\widetilde{K}}_{x}, 1\right) \leq(1 / k) L_{\beta}\left(\widetilde{\widetilde{K}}_{x}, U_{\beta} 1\right)=(1 / k) \widetilde{\widetilde{K}} 1(x)<\infty$. Therefore, since $E$ satisfies (2.2.a), there exists a measure $\mu_{x}$ on $(E, \mathcal{B})$ such that $\widetilde{\widetilde{K}}_{x}=\mu_{x} \circ U_{\beta}$. We define the kernel $\widetilde{K}:$ bp $\mathcal{B} \rightarrow \mathrm{pB}_{F}$ by $\widetilde{K} f(x)=\mu_{x}(f)$. Hence $\widetilde{K}\left(U_{\beta} f\right)(x)=$ $\mu_{x} \circ U_{\beta}(f)=\widetilde{\widetilde{K}} f(x)$ for all $x \in E$; therefore, the function $x \mapsto \mu_{x}(f)$ is $\mathcal{B}_{F}$-measurable for all $f \in \operatorname{bp} \mathcal{B}$ and we clearly have $\widetilde{K} U_{\beta} f=K U_{\beta} f$, $\gamma$-a.e.

Proposition 2.2. If $v: E \rightarrow \mathbb{R}_{+}$and $\beta>0$, then the following assertions are equivalent:

(i) $v \in \mathcal{E}\left(\mathcal{U}_{\beta}\right)$.

(ii) For every continuous increasing concave function $\varphi: I \rightarrow \mathbb{R}_{+}$, where $I$ is an interval such that $\operatorname{Im}(v) \subset I$, it follows that $\varphi \circ v \in \mathcal{E}\left(\mathcal{U}_{\beta}\right)$.

(iii) $1-e^{-\alpha v} \in \mathcal{E}\left(\mathcal{U}_{\beta}\right)$ for all $\alpha>0$.

(iv) There exists a sequence $\left(\alpha_{n}\right)_{n} \subset \mathbb{R}_{+}^{*}, \alpha_{n} \searrow 0$, such that $1-e^{-\alpha_{n} v} \in \mathcal{E}\left(\mathcal{U}_{\beta}\right)$.

Proof. (i) $\Rightarrow$ (ii). By the Jensen inequality we have $\alpha U_{\beta+\alpha}(\varphi \circ v)(x) \leq \varphi\left(\alpha U_{\beta+\alpha} v(x)\right) \leq$ $\varphi(v(x))$, hence $\varphi \circ v$ is $\mathcal{U}_{\beta}$-supermedian. Since $\varphi$ is continuous, $\varphi \circ v$ is finely continuous and therefore $\varphi \circ v \in \mathcal{E}\left(\mathcal{U}_{\beta}\right)$.

(ii) $\Rightarrow$ (iii). The implication follows since the function $\varphi: \mathbb{R}_{+} \rightarrow \mathbb{R}_{+}$defined by $\varphi(x)=1-e^{-\alpha x}$ is continuous, concave and increasing.

(iv) $\Rightarrow$ (i). Let $v_{n}:=\left(1-e^{-\alpha_{n} v}\right) / \alpha_{n}$. By hypothesis, $\left(v_{n}\right)_{n}$ is an increasing sequence from $\mathcal{E}\left(\mathcal{U}_{\beta}\right)$, hence $v=\sup _{n} v_{n}$ is also $\mathcal{U}_{\beta}$-excessive.

For the next corollary we do not assume that $D_{\mathcal{U}_{\beta}}=E$. If $\mathcal{F}$ is a convex cone of real valued functions, we shall denote by $[\mathcal{F}]$ the vector space spanned by $\mathcal{F}:[\mathcal{F}]=\{u-v \mid$ $u, v \in \mathcal{F}\}$.

Corollary 2.3 (cf. [18]).

(1) Let $v: E \rightarrow \mathbb{R}_{+}$and let $\varphi: I \rightarrow \mathbb{R}_{+}$be an increasing concave function, where $I$ is an interval such that $\operatorname{Im}(v) \subset$ I. If $v \in \mathcal{S}\left(\mathcal{U}_{\beta}\right)$ then $\varphi \circ v \in \mathcal{S}\left(\mathcal{U}_{\beta}\right)$. In particular, the vector space $\left[\mathrm{bS}\left(\mathcal{U}_{\beta}\right)\right]$ is an algebra and $v \in \mathcal{S}\left(\mathcal{U}_{\beta}\right)$ if and only if $1-e^{-\alpha v} \in \mathcal{S}\left(\mathcal{U}_{\beta}\right)$ for all $\alpha>0$.

(2) The following assertions are equivalent:

(i) $D_{\mathcal{U}_{\beta}}=E$.

(ii) The vector space $\left[\mathrm{b} \mathcal{E}\left(\mathcal{U}_{\beta}\right)\right]$ is a unitary algebra.

Proof. (1) The first assertion follows by the Jensen inequality as in the proof of Proposition 2.2. the implication (i) $\Rightarrow$ (ii). To prove that $\left[\mathrm{b} \mathcal{S}\left(\mathcal{U}_{\beta}\right)\right]$ is an algebra, it suffices to show that $v^{2} \in\left[\mathrm{b} \mathcal{S}\left(\mathcal{U}_{\beta}\right)\right]$ for every $v \in \mathrm{b} \mathcal{S}\left(\mathcal{U}_{\beta}\right)$. We may assume that $v \leq 1$ and let $\varphi:[0,1] \rightarrow \mathbb{R}_{+}$be defined by $\varphi(x)=2 x-x^{2}$. Then $\varphi$ is concave and increasing, hence 
$\varphi \circ v \in \mathrm{b} \mathcal{S}\left(\mathcal{U}_{\beta}\right)$, and therefore $v^{2} \in\left[\mathrm{b} \mathcal{S}\left(\mathcal{U}_{\beta}\right)\right]$. The last assertion follows again as in the proof of Proposition 2.2

(2) The implication (i) $\Rightarrow$ (ii) follows from Proposition 2.2 and the above considerations.

(ii) $\Rightarrow$ (i). Let $\mathcal{A}$ be the closure of $\left[\mathrm{b} \mathcal{E}\left(\mathcal{U}_{\beta}\right)\right]$ in the supremum norm. It is a Banach algebra, and therefore a lattice with respect to the pointwise order relation. Since $\lim _{\alpha \rightarrow \infty} \alpha U_{\beta+\alpha} v=v$ pointwise for all $v \in \mathcal{E}\left(\mathcal{U}_{\beta}\right)$, the same property holds for all $v \in \mathcal{A}$. Consequently, since $1 \in \mathcal{A}$, we have $\widehat{1}=1$ and if $u_{1}, u_{2} \in \mathcal{E}\left(\mathcal{U}_{\beta}\right)$ then the $\mathcal{U}_{\beta}$ supermedian function $v=\inf \left(u_{1}, u_{2}\right)$ belongs to $\mathcal{A}$, and therefore $\widehat{v}=v, D_{\mathcal{U}_{\beta}}=E$.

Recall that a function $\varphi: \mathcal{S} \rightarrow \mathbb{R}$ is called positive definite if for all $n \geq 1$, $\left\{v_{1}, \ldots, v_{n}\right\} \subset \mathcal{S}$ and $\left\{a_{1}, \ldots, a_{n}\right\} \subset \mathbb{R}$ we have

$$
\sum_{i, j} a_{i} a_{j} \varphi\left(v_{i}+v_{j}\right) \geq 0 .
$$

A function $\varphi: \mathcal{S} \rightarrow \mathbb{R}$ is called negative definite provided that for all $n \geq 2$, $\left\{v_{1}, \ldots, v_{n}\right\} \subset \mathcal{S}$ and $\left\{a_{1}, \ldots, a_{n}\right\} \subset \mathbb{R}$ with $\sum_{i=1}^{n} a_{i}=0$ we have

$$
\sum_{i, j} a_{i} a_{j} \varphi\left(v_{i}+v_{j}\right) \leq 0 .
$$

Considering $\mathcal{S}$ as an Abelian semigroup, a bounded semicharacter of $\mathcal{S}$ is a function $\rho: \mathcal{S} \rightarrow[-1,1]$ such that $\rho(0)=1$ and $\rho(u+v)=\rho(u) \rho(v)$ for all $u, v \in \mathcal{S}$. The set $\widehat{\mathcal{S}}$ of all bounded semicharacters of $\mathcal{S}$ is an Abelian semigroup (under pointwise multiplication, with neutral element the constant semicharacter 1) and it also is a compact Hausdorff topological semigroup endowed with the topology of pointwise convergence.

The folowing two results hold for positive and negative definite functions on $\mathcal{S}$ (cf. [1] and [13]).

(2.6) Let $\Psi: \mathcal{S} \rightarrow \mathbb{R}$. Then $\Psi$ is negative definite if and only if $e^{-t \Psi}$ is positive definite for all $t>0$.

(2.7) Let $\varphi: \mathcal{S} \rightarrow \mathbb{R}$ be a bounded positive definite function. Then there exists a unique positive Radon measure $v$ on $\widehat{\mathcal{S}}$ such that

$$
\varphi(v)=\int_{\widehat{\mathcal{S}}} \rho(v) v(d \rho) \quad \text { for all } v \in \mathcal{S} .
$$

The following result will be a main tool in proving that the space of measures $M(E)$ satisfies (2.2.a) with respect to the resolvent associated with the semigroup $\left(Q_{t}\right)_{t \geq 0}$.

Recall that the specific order relation on $\mathcal{S}$ is denoted by $\prec$ and is defined as follows: if $u, v \in \mathcal{S}$ then

$$
u \prec v \Leftrightarrow \text { there exists } w \in \mathcal{S} \text { such that } u+w=v \text {. }
$$

Proposition 2.4. Assume that $E$ satisfies (2.2.a) and there exists $k>0$ such that $U_{\beta} 1 \geq k$. Let $\varphi: \mathcal{S} \rightarrow[0,1]$ be a positive definite function having the following two order continuity properties: 
(i) If $v \in \mathcal{S}$ then $\varphi\left(\frac{1}{n} v\right) \nearrow \varphi(0)$.

(ii) If $\left(v_{n}\right)_{n} \subset \mathcal{S}$ is pointwise increasing to $v \in \mathcal{S}$ then $\varphi\left(v_{n}\right) \searrow \varphi(v)$.

Then there exists a unique finite measure $\bar{P}$ on $(M(E), \mathcal{M}(E))$ such that

$$
\varphi(v)=\bar{P}\left(e_{v}\right) \quad \text { for all } v \in \mathcal{S} .
$$

Proof. By (2.7) there exists a Radon measure $\gamma$ on $\widehat{\mathcal{S}}$ such that $\varphi(v)=\int_{\widehat{\mathcal{S}}} \rho(v) \gamma(d \rho)$ for all $v \in \mathcal{S}$. Observe first that if $v \in \mathcal{S}$ is such that $\varphi(v)=0$, then $\varphi$ is identically zero. Indeed, it follows from $0=\varphi(v)=\int_{\widehat{\mathcal{S}}} \rho(v) \gamma(d \rho)$ that the function $\rho \mapsto \rho(v)$ vanishes $\gamma$-a.e., and therefore

$$
0=\int_{\widehat{\mathcal{S}}} \rho(v)^{1 / n} \gamma(d \rho)=\int_{\widehat{\mathcal{S}}} \rho\left(\frac{1}{n} v\right) \gamma(d \rho)=\varphi\left(\frac{1}{n} v\right) .
$$

By property (i) we see that $\varphi(0)=\sup _{n} \varphi\left(\frac{1}{n} v\right)=0$ and since $\varphi$ is bounded we conclude that $\varphi(u) \leq \varphi(0)$ for all $u \in \mathcal{S}$, hence $\varphi \equiv 0$.

Assume further that $\varphi \neq 0$. Then

$$
0 \neq \gamma(\widehat{\mathcal{S}})=\varphi(0)=\sup _{n} \varphi\left(\frac{1}{n}\right)=\int_{\widehat{\mathcal{S}}} \sup _{n} \rho(1)^{1 / n} \gamma(d \rho)=\gamma([\rho(1)>0]) .
$$

Let $\widehat{\mathcal{S}}_{+}:=[\rho(1)>0]$. By the above considerations we have $\gamma\left(\widehat{S} \backslash \widehat{\mathcal{S}}_{+}\right)=0$ and $\rho(v)>0$ for every $\rho \in \widehat{\mathcal{S}}_{+}$and $v \in \mathcal{S}$.

Define the function $k: \dot{\mathcal{S}} \times \mathcal{S} \rightarrow \mathbb{R}_{+}$by

$$
k(\rho, v)=\left\{\begin{array}{lr}
-\ln \rho(v) & \text { if } \rho \in \widehat{\mathcal{S}}_{+}, \\
0 & \text { otherwise. }
\end{array}\right.
$$

Since the function $\rho \mapsto k(\rho, v)$ is the extension by zero on $\widehat{\mathcal{S}} \backslash \widehat{\mathcal{S}}_{+}$of a continuous function defined on the open set $\widehat{\mathcal{S}}_{+}$, we deduce that it is $\mathcal{B}(\widehat{\mathcal{S}})$-measurable. Consequently, we may define a mapping $K: \mathcal{S} \rightarrow \mathrm{p} \mathcal{B}(\widehat{\mathcal{S}})$ by $K v:=k(\cdot, v)$. We claim that $K$ satisfies the hypothesis of Lemma 2.1. The positive functional $v \mapsto k(\rho, v)$ is additive and homogeneous over the positive rational numbers. Notice that every positive definite function on $\mathcal{S}$ is decreasing with respect to the specific order on $\mathcal{S}$. In particular, if $u, v \in \mathcal{S}$ and $u \prec v$ then $k(\rho, u) \leq k(\rho, v)$. Let now $\left(u_{n}\right)_{n} \subset \mathcal{S}$ be such that $\sum_{n} u_{n}=v \in \mathcal{S}$. If we put $v_{n}=\sum_{i \leq n} u_{i}$, then by (ii) we get $\varphi\left(v_{n}\right) \searrow \varphi(v)$, or equivalently $\int_{\widehat{S}}\left[\rho(v)-\rho\left(v_{n}\right)\right] \gamma(d \rho) \searrow 0$. Hence $\gamma$-a.e. (in $\left.\rho\right): \rho\left(v_{n}\right) \searrow \rho(v)$ and $\sum_{n} k\left(\rho, u_{n}\right) \stackrel{=}{=} \lim _{n} k\left(\rho, v_{n}\right)=k(\rho, v)=\sum_{n} K u_{n}(\rho)=K v(\rho)$. We conclude that there exists a kernel $\widetilde{K}: \mathrm{bp} \mathcal{B} \rightarrow \mathrm{p} \mathcal{B}(\widehat{\mathcal{S}})$ such that for all $v \in \mathcal{S}, v=U_{\beta} f$ with $f \in$ bp $\mathcal{B}$ we have $\gamma([K v \neq \widetilde{K} v])=0$. We remark that $\widetilde{K} 1 \widetilde{K}^{<}<\gamma$-a.e. and if $\rho \in[\widetilde{K} 1<\underset{\widetilde{K}}{]}]=: \widehat{\mathcal{S}}_{1}$ then the functional $f \mapsto \widetilde{K}_{\rho}(f):=\widetilde{K} f(\rho)$ is given by a finite measure $\widetilde{K}_{\rho} \in M(E)$.

We define the measure $\bar{P}$ on $(M(E), \mathcal{M}(E))$ by

$$
\int_{M(E)} F(\mu) \bar{P}(d \mu):=\int_{\widehat{\mathcal{S}}_{1}} F\left(\widetilde{K}_{\rho}\right) \gamma(d \rho), \quad F \in \operatorname{bp} \mathcal{M}(E) .
$$


If $v=U_{\beta} f$ then $\bar{P}\left(e_{v}\right)=\int_{\widehat{\mathcal{S}}_{1}} e_{v}\left(\widetilde{K}_{\rho}\right) \gamma(d \rho)=\int_{\widehat{\mathcal{S}}_{1}} e^{-\widetilde{K} v(\rho)} \gamma(d \rho)=\int_{\widehat{\mathcal{S}}_{+}} e^{-k(\rho, v)} \gamma(d \rho)$ $=\int_{\widehat{\mathcal{S}}_{+}} \rho(v) \gamma(d \rho)=\varphi(v)$. If $v \in \mathcal{S}$ then there exists a sequence $\left(f_{n}\right)_{n} \subset$ bp $\mathcal{B}$ such that $U_{\beta} f_{n} \nearrow v$ and by (ii) we get $\varphi\left(U_{\beta} f_{n}\right) \searrow \varphi(v)$. Since clearly $\bar{P}\left(e_{U_{\beta} f_{n}}\right) \searrow \bar{P}\left(e_{v}\right)$, we conclude that $\varphi(v)=\bar{P}\left(e_{v}\right)$ for all $v \in \mathcal{S}$.

The uniqueness of $\bar{P}$ follows by a monotone class argument because the vector space spanned by $\left\{e_{v} \mid v \in \mathcal{S}\right\}$ is an algebra of functions on $M(E)$ generating the $\sigma$-algebra $\mathcal{M}(E)$.

Remark 2.5. Let $\varphi: \mathcal{S} \rightarrow[0,1]$ be a positive definite function such that if $\left(v_{n}\right)_{n} \subset \mathcal{S}$ is specifically decreasing to zero (i.e., decreasing to zero with respect to the specific order relation on $\mathcal{S})$, then $\varphi\left(v_{n}\right) \nearrow \varphi(0)$. For every $\left(u_{n}\right)_{n} \subset \mathcal{S}$ which is specifically increasing to $u \in \mathcal{S}$ we have $\varphi\left(u_{n}\right) \searrow \varphi(u)$.

The assertion follows from the inequality

$$
\varphi(u)+\varphi(v) \leq \varphi(0)+\varphi(u+v) \quad \text { for all } u, v \in \mathcal{S},
$$

since $0 \leq \varphi\left(u_{n}\right)-\varphi(u) \leq \varphi(0)-\varphi\left(u-u_{n}\right)$ and the sequence $\left(u-u_{n}\right)_{n}$ is specifically decreasing to zero.

Corollary 2.6 ([13, Corollary (A.6)]). Let $\varphi: \mathrm{bp} \mathcal{B} \rightarrow[0,1]$ be positive definite such that $\varphi\left(f_{n}\right) \nearrow \varphi(0)$ whenever $\left(f_{n}\right)_{n} \subset \mathrm{bp} \mathcal{B}$ and $f_{n} \searrow 0$ pointwise. Then there exists a unique finite measure $\bar{P}$ on $(M(E), \mathcal{M}(E))$ such that

$$
\varphi(f)=\bar{P}\left(e_{f}\right) \quad \text { for all } f \in \mathrm{bp} \mathcal{B} .
$$

Proof. The assertion follows from Proposition 2.4 applied to the resolvent of kernels $\mathcal{U}=\left(\frac{1}{1+\alpha} I\right)_{\alpha>0}$ and from Remark 2.5 , because $\mathcal{E}\left(\mathcal{U}_{\beta}\right)=\mathrm{p} \mathcal{B}$ in this case.

\section{The nonlinear semigroup}

We assume in this section that $\mathcal{U}=\left(U_{\alpha}\right)_{\alpha>0}$ is the resolvent of a right process $X$ with state space $E$. Recall that if $\beta>0$ then the $\beta$-subprocess of $X$ has the transition semigroup $\left(P_{t}^{\beta}\right)_{t \geq 0}$, where $P_{t}^{\beta}=e^{-\beta t} P_{t}$ and its resolvent is precisely $\mathcal{U}_{\beta}=\left(U_{\beta+\alpha}\right)_{\alpha>0}$. We suppose that $P_{t} 1=1$.

Let us put

$$
\Phi_{\beta}(x, \lambda)=\Phi(x, \lambda)+\beta \lambda, \quad x \in E, \lambda \geq 0,
$$

and for each $f \in \mathrm{bp} \mathcal{B}$ consider the equation

$$
V_{t} f(x)=P_{t}^{\beta} f(x)+\int_{0}^{t} P_{s}^{\beta}\left(x, \Phi_{\beta}\left(\cdot, V_{t-s} f\right)\right) d s, \quad t \geq 0, x \in E .
$$

If $A$ denotes the infinitesimal generator of $X$, then equation 3.1 is formally equivalent to

$$
\left\{\begin{array}{l}
\frac{d}{d t} v_{t}(x)=A v_{t}(x)+\Phi\left(x, v_{t}(x)\right) \\
v_{0}=f
\end{array}\right.
$$


Since the infinitesimal generator of the $\beta$-subprocess of $X$ is $A-\beta$, it is again formally clear that equations (1.1) and (3.1) are equivalent. The precise result is given by the following proposition. The idea of its proof has been suggested to us by Habib Maagli (private communication).

Proposition 3.1. Let $f \in \mathrm{bp} \mathcal{B}$ and let $(t, x) \mapsto v_{t}(x)$ be a jointly measurable function such that $\sup _{0 \leq s \leq t}\left\|v_{s}\right\|_{\infty}<\infty$ for all $t>0$. Then $v_{t}$ is a solution of (1.1) if and only if it is a solution of (3.1.

We shall use the notation

$$
\beta:=\left\|b^{-}\right\|_{\infty} .
$$

The following result is essentially Proposition 2.3 from [13] stated there without proof. However, as mentioned in Introduction, we had access to a manuscript of its proof. Since the method of proof is important for our approach, we shall outline it in the Appendix.

Proposition 3.2. (i) For every $f \in \mathrm{bp} \mathcal{B}$ equation 1.1 has a unique solution $(t, x) \mapsto$ $V_{t} f(x)$ jointly measurable in $(t, x)$ such that $\sup _{0 \leq s \leq t}\left\|V_{s} f\right\|_{\infty}<\infty$ for all $t>0$.

(ii) For all $t \geq 0$ and $x \in E$ we have $0 \leq V_{t} f(x) \leq e^{\bar{\beta} t}\|f\|_{\infty}$.

(iii) If $t \mapsto P_{t} f(x)$ is right continuous on $[0, \infty)$ for all $x \in E$, then so is $t \mapsto V_{t} f(x)$.

(iv) The mappings $f \mapsto V_{t} f$ form a nonlinear semigroup of operators on bp $\mathcal{B}$.

(v) For all $t \geq 0$ and $\mu \in M(E)$ the map $f \mapsto\left\langle\mu, V_{t} f\right\rangle$ is negative definite on the semigroup bp $\mathcal{B}$.

(vi) If $\left(f_{n}\right)_{n} \subset \mathrm{bp} \mathcal{B}$ is a decreasing sequence with $f_{n} \searrow f$, then $V_{t} f_{n} \searrow V_{t} f$ for every $t \geq 0$.

The next result is a version of Proposition 2.7 from [13].

Proposition 3.3. If $b$ is a bounded, $\mathcal{B}$-measurable function and $t \geq 0$, then the following assertions hold.

(i) For every $f \in \mathrm{bp} \mathcal{B}$ the equation

$$
k_{t}=P_{t} f-\int_{0}^{t} P_{s}\left(b k_{t-s}\right) d s
$$

has a unique solution $(t, x) \mapsto P_{t}^{b} f(x)$ jointly measurable in $(t, x)$ such that $\sup _{0 \leq s \leq t}\left\|P_{s} f\right\|_{\infty}<\infty$ for all $t>0$. The family $\left(P_{t}^{b}\right)_{t \geq 0}$ is a semigroup of bounded kernels on $(E, \mathcal{B})$ and $e^{-\beta^{\prime} t} P_{t} \leq P_{t}^{b} \leq e^{\beta t} P_{t}$, where $\beta^{\prime}:=\left\|b^{+}\right\|_{\infty}$. More precisely, for every $x \in E$ we have

$$
P_{t}^{b} f(x)=E^{x}\left(e^{-\int_{0}^{t} b\left(X_{s}\right) d s} f\left(X_{t}\right)\right) .
$$

(ii) If $b \geq 0$ then the semigroup $\left(P_{t}^{b}\right)_{t \geq 0}$ is sub-Markovian, $P_{t}^{b} \leq P_{t}$ and all the points of $E$ are non-branch points for $\left(P_{t}^{\bar{b}}\right)_{t \geq 0}$, i.e., with respect to the resolvent $\mathcal{U}_{b}$ generated by $\left(P_{t}^{b}\right)_{t \geq 0}$. The fine topologies on $E$ generated by $\mathcal{U}$ and $\mathcal{U}_{b}$ coincide.

(iii) If $\alpha \in \mathbb{R}$ then $P_{t}^{b+\alpha}=e^{-\alpha t} P_{t}^{b}$. If $b_{1}$ and $b_{2}$ are two bounded, $\mathcal{B}$-measurable functions and $b_{1} \leq b_{2}$, then

$$
\left(P_{t}^{b_{2}-b_{1}}\right)^{b_{1}}=P_{t}^{b_{2}} \leq P_{t}^{b_{1}} .
$$




\section{The measure-valued right process}

The transition function on the space of measures and a key property are given by the next two results which are due to P. J. Fitzsimmons, [13]. For the reader's convenience we present their proofs in the Appendix.

Let $\left(V_{t}\right)_{t \geq 0}$ be the nonlinear semigroup of operators on bp $\mathcal{B}$ given by Proposition 3.2

Proposition 4.1. There exists a unique Markovian semigroup of kernels $\left(Q_{t}\right)_{t \geq 0}$ on $(M(E), \mathcal{M}(E))$ such that

$$
Q_{t}\left(e_{f}\right)=e_{V_{t} f} \quad \text { for every } f \in \mathrm{bp} \mathcal{B} \text { and } t>0 .
$$

Proposition 4.2. If $f \in \mathrm{bp} \mathcal{B}$ and $t>0$, then

$$
Q_{t}\left(l_{f}\right)=l_{P_{t}^{b} f} .
$$

Let $\overline{\mathcal{U}}=\left(\bar{U}_{\alpha}\right)_{\alpha>0}$ be the Markovian resolvent of kernels on $(M(E), \mathcal{M}(E))$ generated by the semigroup $\left(Q_{t}\right)_{t \geq 0}$ given by Proposition 4.1 .

Recall that $\beta=\left\|b^{-}\right\|_{\infty}$, let $\beta^{\prime} \geq \beta$ and

$$
b^{\prime}:=b+\beta^{\prime} .
$$

Then $b^{\prime} \geq 0$ and by assertion (ii) of Proposition 3.3 the resolvent $\mathcal{U}_{b^{\prime}}$ generated by $\left(P_{t}^{b^{\prime}}\right)_{t \geq 0}$ is sub-Markovian and bounded if $\beta^{\prime}>\beta$.

Corollary 4.3. If $u \in \mathrm{bp} \mathcal{B}$ then the following assertions are equivalent:

(i) $u \in \mathcal{E}\left(\mathcal{U}_{b^{\prime}}\right)$.

(ii) $l_{u} \in \mathcal{E}\left(\overline{\mathcal{U}}_{\beta^{\prime}}\right)$.

(iii) For every $\alpha>0$ we have $1-e_{\alpha u} \in \mathcal{E}\left(\overline{\mathcal{U}}_{\beta^{\prime}}\right)$.

Proof. (i) $\Leftrightarrow$ (ii). Since by Proposition 3.3 we have $P_{t}^{b^{\prime}}=e^{-\beta^{\prime} t} P_{t}^{b}$, we deduce from Proposition 4.2 that

$$
e^{-\beta^{\prime} t} Q_{t}\left(l_{u}\right)=l_{P_{t}^{b^{\prime} u}},
$$

and therefore $u \in \mathcal{S}\left(\mathcal{U}_{b^{\prime}}\right)$ if and only if $l_{u} \in \mathcal{S}\left(\overline{\mathcal{U}}_{\beta^{\prime}}\right)$. We also deduce that

$$
u=\lim _{t \searrow 0} P_{t}^{b^{\prime}} u \Leftrightarrow l_{u}=\lim _{t \searrow 0} l_{P_{t}^{b^{\prime}} u},
$$

thus the claimed equivalence holds.

The equivalence (ii) $\Leftrightarrow$ (iii) follows by the last part of Corollary 2.3 1 ), using Propositions 3.2 (iii) and 4.1 .

Corollary 4.4. If $t>0$ and $u \in \mathrm{b} \mathcal{E}\left(\mathcal{U}_{b^{\prime}}\right)$, then $1-e_{V_{t} u} \in \mathcal{E}\left(\overline{\mathcal{U}}_{\beta^{\prime}}\right)$.

Proof. According to Corollary 4.3 , we have $1-e_{u} \in \mathcal{E}\left(\overline{\mathcal{U}}_{\beta^{\prime}}\right)$, and therefore $1-e_{V_{t} u}=$ $Q_{t}\left(1-e_{u}\right) \in \mathcal{E}\left(\overline{\mathcal{U}}_{\beta^{\prime}}\right)$. 
Proposition 4.5. All the points of $M(E)$ are non-branch points for the semigroup $\left(Q_{t}\right)_{t \geq 0}$ (i.e. with respect to the resolvent $\overline{\mathcal{U}}_{\beta^{\prime}}$ ).

Proof. According to Corollary 3.6 from [18], it will be sufficient to prove that the uniqueness of charges and the specific solidity of potentials hold for $\overline{\mathcal{U}}_{\beta^{\prime}}$.

1. The uniqueness of charges. Assume that $\beta^{\prime}>\beta$. We have to show that if $\mu, v$ are two finite measures on $(M(E), \mathcal{M}(E))$ such that $\mu \circ \bar{U}_{\beta^{\prime}}=v \circ \bar{U}_{\beta^{\prime}}$, then $\mu=v$. Indeed, from $\mu \circ \bar{U}_{\beta^{\prime}}=v \circ \bar{U}_{\beta^{\prime}}$ we see that $\mu(F)=v(F)$ for every $F \in \mathcal{E}\left(\overline{\mathcal{U}}_{\beta^{\prime}}\right)$. It follows by Corollary 4.3 that the above equality holds for every $F$ lying in the linear space spanned by $\left\{e_{u} \mid u \in \mathrm{b} \mathcal{E}\left(\mathcal{U}_{b^{\prime}}\right)\right\}$. Since this space is an algebra of bounded $\mathcal{M}(E)$-measurable functions and generates $\mathcal{M}(E)$, by a monotone class argument we conclude that $\mu=\nu$.

2. The specific solidity of potentials. We have to show that if $\xi, \mu \circ \bar{U}_{\beta^{\prime}} \in \operatorname{Exc}\left(\overline{\mathcal{U}}_{\beta^{\prime}}\right)$ and $\xi \prec \mu \circ \bar{U}_{\beta^{\prime}}$, then $\xi$ is a potential; here $\prec$ denotes the specific order relation on $\operatorname{Exc}\left(\overline{\mathcal{U}}_{\beta^{\prime}}\right)$. Let $\mathcal{A}$ be the additive semigroup generated by $\left\{V_{t} u \mid u \in \mathrm{b} \mathcal{E}\left(\mathcal{U}_{b^{\prime}}\right), t \geq 0\right\}$ and $[\overline{\mathcal{A}}]$ the vector space spanned by $\left\{e_{v} \mid v \in \mathcal{A}\right\}$. Then $[\overline{\mathcal{A}}]$ clearly is an algebra, $1 \in[\overline{\mathcal{A}}]$ and since $\left\{e_{u} \mid u \in \mathrm{b} \mathcal{E}\left(\mathcal{U}_{b^{\prime}}\right)\right\} \subset[\overline{\mathcal{A}}]$ we have $\sigma([\overline{\mathcal{A}}])=\mathcal{M}(E)$. We show that $[\overline{\mathcal{A}}] \subset\left[\mathrm{b} \mathcal{E}\left(\overline{\mathcal{U}}_{\beta^{\prime}}\right)\right]$. Corollary 4.4 implies that $1-e_{V_{t} u} \in \mathrm{b} \mathcal{E}\left(\overline{\mathcal{U}}_{\beta^{\prime}}\right)$ provided that $u \in \mathrm{b} \mathcal{E}\left(\mathcal{U}_{b^{\prime}}\right)$. Since by Corollary 2.3. $\left[\mathrm{b} \mathcal{S}\left(\overline{\mathcal{U}}_{\beta^{\prime}}\right)\right]$ is an algebra, $e_{v} \in\left[\mathrm{b} \mathcal{S}\left(\overline{\mathcal{U}}_{\beta^{\prime}}\right)\right]$ for every $v \in \mathcal{A}$. So we have to prove that the map $s \mapsto Q_{s}\left(e_{v}\right)(\mu)$ is right continuous on $[0, \infty)$ for every $v \in \mathcal{A}$ and $\mu \in M(E)$. According to Proposition 4.1 we have $Q_{s}\left(e_{v}\right)=e_{V_{s} v}$ and therefore, by Proposition 3.2(iii), it will be sufficient to show that the map $s \mapsto P_{s} v(x)$ is right continuous for every $v \in \mathcal{A}$ and $x \in E$. We may assume that $v=V_{t} u$ with $u \in \mathrm{b} \mathcal{E}\left(\mathcal{U}_{b^{\prime}}\right)$ and $t \geq 0$. We have

$$
V_{s+t} u=P_{s} V_{t} u+\int_{0}^{s} P_{\alpha} \Phi\left(\cdot, V_{s+t-\alpha} u\right) d \alpha .
$$

Again by Proposition 3.2 we know that $s \mapsto V_{s+t} u$ is right continuous and thus $s \mapsto$ $\int_{0}^{s} P_{\alpha} \Phi\left(\cdot, V_{s+t-\alpha} u\right) d \alpha$ is also right continuous (by dominated convergence), concluding that $s \mapsto P_{s} V_{t} u$ has the same property.

Let $\xi, \mu \circ \bar{U}_{\beta^{\prime}} \in \operatorname{Exc}\left(\overline{\mathcal{U}}_{\beta^{\prime}}\right)$ with $\xi \prec \mu \circ \bar{U}_{\beta^{\prime}}$. We may suppose that $\mu(1) \leq 1$. Indeed, if it is not the case, then $\mu=\sum_{n} \mu_{n}$ with $\mu_{n}$ (1) $\leq 1$ for all $n$ and by Ch. 2 in [2] there exists a sequence $\left(\xi_{n}\right)_{n} \subset \operatorname{Exc}\left(\overline{\mathcal{U}}_{\beta^{\prime}}\right)$ such that $\xi=\sum_{n} \xi_{n}$ and $\xi_{n} \prec \mu_{n} \circ \bar{U}_{\beta}$ for every $n$. Let $\varphi_{\xi}: \mathcal{E}\left(\overline{\mathcal{U}}_{\beta^{\prime}}\right) \rightarrow \overline{\mathbb{R}}_{+}$be the functional defined by $\varphi_{\xi}(F)=\bar{L}_{\beta^{\prime}}(\xi, F)$ for $F \in \mathcal{E}\left(\overline{\mathcal{U}}_{\beta^{\prime}}\right)$, where $\bar{L}_{\beta^{\prime}}$ denotes the energy functional associated with $\overline{\mathcal{U}}_{\beta^{\prime}}$.

By the first part of the proof, we may extend $\varphi_{\xi}$ to an increasing linear functional on $[\overline{\mathcal{A}}]$. Let $\mathcal{L}$ be the closure of $[\overline{\mathcal{A}}]$ with respect to the sup norm. Clearly, $\mathcal{L}$ is a vector lattice and we claim that $\varphi_{\xi}$ extends to a positive linear functional on $\mathcal{L}$. Indeed, if $\left(F_{n}\right)_{n} \subset[\overline{\mathcal{A}}]$ is a sequence converging uniformly to zero and we consider a sequence $\left(v_{k} \circ \bar{U}_{\beta^{\prime}}\right)_{k} \subset$ $\operatorname{Pot}\left(\overline{\mathcal{U}}_{\beta^{\prime}}\right)$ with $v_{k} \circ \bar{U}_{\beta^{\prime}} \nearrow \xi$, then

$\left|\varphi_{\xi}\left(F_{n}\right)\right|=\lim _{k}\left|v_{k}\left(F_{n}\right)\right| \leq \liminf _{k} v_{k}\left(\left|F_{n}\right|\right) \leq \varepsilon \liminf _{k} v_{k}(1)=\varepsilon \bar{L}_{\beta^{\prime}}(\xi, 1) \leq \varepsilon \mu(1) \leq \varepsilon$, provided that $n \geq n_{0}$ and $\left\|F_{n}\right\|_{\infty}<\varepsilon$ for all $n \geq n_{0}$. 
Since $\xi \prec \mu \circ \bar{U}_{\beta^{\prime}}$ we have $\varphi_{\xi}(F) \leq \mu(F)$ for every $F \in \mathcal{L}_{+}$, and therefore, if $\left(F_{n}\right)_{n} \subset \mathcal{L}_{+}$is a sequence decreasing pointwise to zero, then $\varphi_{\xi}\left(F_{n}\right) \searrow 0$. By the Daniell theorem there exists a measure $v$ on $(M(E), \mathcal{M}(E))$ such that $\varphi_{\xi}(F)=v(F)$ for all $F \in \mathcal{L}$. In particular, if $u \in \mathrm{b} \mathcal{E}\left(\mathcal{U}_{b^{\prime}}\right)$ then $\bar{L}_{\beta^{\prime}}\left(\xi, Q_{t}\left(e_{u}\right)\right)=\varphi_{\xi}\left(e_{V_{t} u}\right)=v\left(Q_{t}\left(e_{u}\right)\right)$ and therefore

$$
\begin{aligned}
\bar{L}_{\beta^{\prime}}\left(\xi, \bar{U}_{\beta^{\prime}}\left(e_{u}\right)\right) & =\lim _{k} v_{k}\left(\bar{U}_{\beta^{\prime}}\left(e_{u}\right)\right)=\int_{0}^{\infty} e^{-\beta^{\prime} t} \lim _{k} v_{k}\left(Q_{t}\left(e_{u}\right)\right) d t \\
& =\int_{0}^{\infty} e^{-\beta^{\prime} t} \bar{L}_{\beta^{\prime}}\left(\xi, Q_{t}\left(e_{u}\right)\right) d t=v\left(\bar{U}_{\beta^{\prime}}\left(e_{u}\right)\right) .
\end{aligned}
$$

We conclude that $\xi=v \circ \bar{U}_{\beta^{\prime}}$.

Remark 4.6. By the last part of the above proof the following assertion holds. If $\xi \in \operatorname{Exc}\left(\overline{\mathcal{U}}_{\beta^{\prime}}\right)$ with $\bar{L}_{\beta^{\prime}}(\xi, 1) \leq 1$ and $v$ is a positive measure on $M(E)$ such that $\bar{L}_{\beta^{\prime}}\left(\xi, Q_{t}\left(e_{u}\right)\right)=v\left(Q_{t}\left(e_{u}\right)\right)$ for all $t>0$ and $u$ lying in a convex cone $\mathcal{C} \subset \mathrm{b} \mathcal{E}\left(\mathcal{U}_{b^{\prime}}\right)$ which is separable in the supremum norm, inf-stable, separates the points of $E$ and there exists a sequence $\left(u_{n}\right)_{n} \subset \mathcal{C}$ with $u_{n} \nearrow 1$, then $\xi=v \circ \bar{U}_{\beta^{\prime}}$.

If $M \in \mathcal{B}$ and $u \in \mathcal{E}\left(\mathcal{U}_{b^{\prime}}\right)$, then recall that the reduced function of $u$ on $M$ (with respect to $\left.\mathcal{U}_{b^{\prime}}\right)$ is the function $R_{b^{\prime}}^{M} u$ defined by

$$
R_{b^{\prime}}^{M} u:=\inf \left\{v \in \mathcal{E}\left(\mathcal{U}_{b^{\prime}}\right) \mid v \geq u \text { on } M\right\}
$$

The reduced function $R_{b^{\prime}}^{M} u$ is universally $\mathcal{B}$-measurable.

We now present a relation between the reduced functions on $E$ and $M(E)$, respectively; we shall denote by $\bar{R}_{\beta^{\prime}}^{\Gamma} F(\Gamma \in \mathcal{M}(E), F \in \mathrm{p} \mathcal{M}(E))$ the reduced function with respect to $\mathcal{E}\left(\overline{\mathcal{U}}_{\beta^{\prime}}\right)$.

Proposition 4.7. If $u \in \mathrm{b} \mathcal{E}\left(\mathcal{U}_{b^{\prime}}\right)$ and $G \in \mathcal{B}$ is a finely open subset of $E$, then

$$
l_{R_{b^{\prime}}^{G} u}=\bar{R}_{\beta^{\prime}}^{M\left(G^{c}\right)^{c}} l_{R_{b^{\prime}}^{G} u} .
$$

Proof. By Theorem 1.3.8 in [2] there exists a sequence $\left(f_{n}\right)_{n} \subset$ bp $\mathcal{B}$ such that $f_{n}=0$ on $G^{c}$ and $\left(U_{b^{\prime}} f_{n}\right)_{n}$ increases to $R_{b^{\prime}}^{G} u$. From Proposition 4.2 we get $\bar{U}_{\beta^{\prime}} l_{f_{n}}=l_{U_{b^{\prime}} f_{n}}$ and since $l_{f_{n}}$ vanishes on $M\left(G^{c}\right)$ we have

$$
l_{R_{b^{\prime}}^{G} u}=\sup _{n} l_{U_{b^{\prime}} f_{n}}=\sup _{n} \bar{U}_{\beta^{\prime}} l_{f_{n}}=\sup _{n} \bar{R}_{\beta^{\prime}}^{M\left(G^{c}\right)^{c}} \bar{U}_{\beta^{\prime}} l_{f_{n}}=\bar{R}_{\beta^{\prime}}^{M\left(G^{c}\right)^{c}} l_{R_{b^{\prime}}^{G} u} .
$$

Assume that $\beta^{\prime}>\beta$. We shall consider the following regularity condition:

(*) $V_{t} v$ belongs to the closure in the supremum norm of $\left[\mathrm{b} \mathcal{E}\left(\mathcal{U}_{b^{\prime}}\right)\right]$ for every $t \geq 0$ and $v$ lying in a convex cone $\mathcal{C} \subset \mathrm{b} \mathcal{E}\left(\mathcal{U}_{b^{\prime}}\right)$ which is separable in the supremum norm, inf-stable, separates the points of $E$ and there exists a sequence $\left(u_{n}\right)_{n} \subset \mathcal{C}$ with $u_{n} \nearrow 1$. 
Proposition 4.8. Each of the following two conditions implies that condition (*) holds:

(4.a) $b, c$ and $N$ do not depend on $x \in E$.

(4.b) $\left(P_{t}\right)_{t \geq 0}$ is a Feller semigroup (on the locally compact space $E$ ) and $V_{t}\left(C_{0}(E)\right) \subset$ $C_{0}(E)$ for every $t \geq 0$.

Proof. By Proposition 2.2 we have $1-e^{-s v} \in \mathrm{b} \mathcal{E}\left(\mathcal{U}_{b^{\prime}}\right)$ provided that $v \in \mathrm{b} \mathcal{E}\left(\mathcal{U}_{b^{\prime}}\right)$ and $s \in \mathbb{R}_{+}$. If (4.a) is satisfied then from the above considerations with the notation from the proof of Proposition 3.2 we have $\varphi(v) \in \mathrm{b} \mathcal{E}\left(\mathcal{U}_{b^{\prime}}\right)$ and, since

$$
V_{t}^{k+1} v=P_{t}^{a} v+\int_{0}^{t} P_{s}^{a} \varphi\left(V_{t-s}^{k} v\right) d s,
$$

we deduce by induction that $V_{t}^{k} v \in \mathrm{b} \mathcal{E}\left(\mathcal{U}_{b^{\prime}}\right)$ for every $t>0$. Since $\left(V_{t}^{k} v(x)\right)_{k}$ is converging uniformly in $x$ to $V_{t} v(x)$ and because the approximation from Step II is also uniform, we conclude that $V_{t}\left(\mathrm{~b} \mathcal{E}\left(\mathcal{U}_{b^{\prime}}\right)\right) \subset \mathrm{b} \mathcal{E}\left(\mathcal{U}_{b^{\prime}}\right)$ and, in particular, condition (*) holds.

If condition (4.b) is satisfied, then there exists a Ray cone $\mathcal{R} \subset \mathrm{b} \mathcal{E}\left(\mathcal{U}_{\beta^{\prime}-\beta}\right)$ such that $\left[\mathcal{R} \cap C_{0}(E)\right]$ is dense in $C_{0}(E)$ in the supremum norm (see e.g. [4]). Condition (*) holds in this case too, because $\mathcal{E}\left(\mathcal{U}_{\beta^{\prime}-\beta}\right) \subset \mathcal{E}\left(\mathcal{U}_{b^{\prime}}\right)$.

Remark. Situations when condition (4.b) is satisfied are presented in [14, Appendix]. Notice that in this case the topology of $E$ is a Ray topology. For other regularity conditions on $\Phi$ and $\left(V_{t}\right)_{t \geq 0}$ see also [12].

Recall that a right process $X$ is called standard if it is quasi-left-continuous on $[0, \zeta)$, i.e., for every increasing sequence $\left(T_{n}\right)_{n}$ of stopping times with limit $T$ we have a.s. $X_{T_{n}} \rightarrow X_{T}$ on $[T<\zeta], \zeta$ being the lifetime of $X$. Notice that since we assumed that $P_{t} 1=1, X$ has infinite lifetime and, clearly, if $X$ is standard, then it is in fact a Hunt process, that is, it is quasi-left-continuous on $[0, \infty)$.

Let $\lambda$ be a finite measure on $E$. An increasing sequence $\left(F_{n}\right)_{n} \subset \mathcal{B}$ is called a $\lambda$-nest provided that

$$
R_{\beta}^{E \backslash F_{n}} 1=0 \quad \lambda \text {-a.e. }
$$

(4.1) The following assertions are equivalent (cf. [3] and [4]).

(4.1.a) There exists a $\lambda$-nest of compact sets.

(4.1.b) There exists a function $v \in \mathcal{E}\left(\mathcal{U}_{\beta}\right) \cap L^{1}(E, \lambda)$ such that for all $n \in \mathbb{N}$ the set $[v \leq n]$ is relatively compact; one says that $v$ has compact level sets.

(4.2) If $X$ has càdlàg trajectories (i.e., it possesses left limits in $E$ a.s. on $[0, \zeta))$ then the above equivalent conditions (4.1.a) and (4.1.b) are satisfied for every finite measure $\lambda$ (see e.g. [2]). Notice that if the topology is a Ray topology and condition (4.1.a) is fulfilled for every $\lambda$, then the process $X$ is standard.

We can now state the main result of this paper.

Theorem 4.9. If condition $(*)$ holds then the following assertions hold.

(i) There exists a Borel right process (called an $(X, \Phi)$-superprocess) with state space $M(E)$ endowed with the weak topology, having $\left(Q_{t}\right)_{t \geq 0}$ as transition semigroup. 
(ii) If $X$ is a Hunt process then the $(X, \Phi)$-superprocess has càdlàg trajectories and if, in addition, $V_{t}(\mathcal{C}) \subset \mathcal{C}$ for all $t>0$, then the $(X, \Phi)$-superprocess is also a Hunt process.

(iii) If $E_{1}$ (resp. $M(E)_{1}$ ) denotes the entrance space of $X$ (resp. the entrance space of the $(X, \Phi)$-superprocess), then $M\left(E_{1}\right) \subset M(E)_{1}$ and the set $M(E)_{1} \backslash M\left(E_{1}\right)$ is polar. In addition, $M\left(E_{1}\right)$ is precisely the saturation of $M(E)$ with respect to $\overline{\mathcal{U}}_{\beta^{\prime}}^{h}$, where $h:=1+l_{1} \in \mathcal{E}\left(\overline{\mathcal{U}}_{\beta^{\prime}}\right)$.

(iv) If $\mu \in M(E), f \in \mathrm{p} \mathcal{B}$ and $G \in \mathcal{B}$ is finely open, then

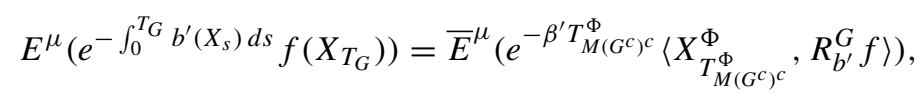

where $T_{G}$ is the hitting time of $G$ by the process $X$, and $T_{M\left(G^{c}\right)^{c}}^{\Phi}$ denotes the hitting time of $M\left(G^{c}\right)^{c}$ by the $(X, \Phi)$-superprocess $\left(X_{t}^{\Phi}\right)_{t \geq 0}$.

Proof. Step I. Assume that $E$ satisfies (2.2.a) with respect to $\mathcal{U}$ and let $\xi \in \operatorname{Exc}\left(\overline{\mathcal{U}}_{\beta^{\prime}}\right)$ be such that $\bar{L}_{\beta^{\prime}}(\xi, h) \leq 1$. Notice that according to Corollary 4.3 we have $l_{u} \in \mathcal{E}\left(\overline{\mathcal{U}}_{\beta^{\prime}}\right)$ and $e_{u} \in\left[\mathrm{b} \mathcal{E}\left(\overline{\mathcal{U}}_{\beta^{\prime}}\right)\right]$ for every $u \in \mathrm{b} \mathcal{E}\left(\mathcal{U}_{b^{\prime}}\right)=: \mathcal{S}$. We define the functional $\varphi: \mathcal{S} \rightarrow \mathbb{R}_{+}$by

$$
\varphi(u):=\bar{L}_{\beta^{\prime}}\left(\xi, e_{u}\right), \quad u \in \mathcal{S} .
$$

We check that $\varphi$ satisfies the conditions from Proposition 2.4. Clearly, $\varphi(u) \in[0,1]$ for every $u \in \mathcal{S}$ since $\varphi(u) \leq \bar{L}_{\beta^{\prime}}(\xi, 1) \leq 1$. Using the inequality $1-e^{-x} \leq x$ if $x \geq 0$, we get

$$
\varphi(0)-\varphi\left(\frac{u}{n}\right)=\bar{L}_{\beta^{\prime}}\left(\xi, 1-e_{u / n}\right) \leq \bar{L}_{\beta^{\prime}}\left(\xi, l_{u / n}\right) \leq \frac{\|u\|_{\infty}}{n} \bar{L}_{\beta^{\prime}}(\xi, 1),
$$

and therefore $\varphi\left(\frac{1}{n} u\right) \nearrow \varphi(0)$. If $\left(u_{n}\right)_{n} \subset \mathcal{S}$ with $u_{n} \nearrow u$ then $\left(1-e_{u_{n}}\right)_{n}$ is a sequence of $\overline{\mathcal{U}}_{\beta^{\prime}}$-excessive functions which is increasing to $1-e_{u}$ and thus

$$
\bar{L}_{\beta^{\prime}}\left(\xi, 1-e_{u_{n}}\right) \nearrow \bar{L}_{\beta^{\prime}}\left(\xi, 1-e_{u}\right),
$$

or equivalently

$$
\varphi\left(u_{n}\right)=\bar{L}_{\beta^{\prime}}\left(\xi, e_{u_{n}}\right) \searrow \bar{L}_{\beta^{\prime}}\left(\xi, e_{u}\right)=\varphi(u) .
$$

It remains to show that $\varphi$ is positive definite. If $\left(a_{i}\right)_{i \leq n} \subset \mathbb{R}$ and $\left(u_{i}\right)_{i \leq n} \subset \mathcal{S}$ then

$$
\sum_{i, j} a_{i} a_{j} \varphi\left(u_{i}+u_{j}\right)=\bar{L}_{\beta^{\prime}}\left(\xi, \sum_{i, j} a_{i} a_{j} e_{u_{i}+u_{j}}\right)=\bar{L}_{\beta^{\prime}}\left(\xi,\left(\sum_{i} a_{i} e_{u_{i}}\right)^{2}\right) \geq 0 .
$$

We conclude that there exists a probability measure $\bar{P}$ on $(M(E), \mathcal{M}(E))$ such that for all $u \in \mathcal{S}$ we have

$$
\varphi(u)=\bar{P}\left(e_{u}\right) .
$$

According to Remark 4.6 in order to show that $\xi=\bar{P} \circ \bar{U}_{\beta^{\prime}}$, it will be sufficient to prove that $\bar{L}_{\beta^{\prime}}\left(\xi, Q_{t}\left(e_{u}\right)\right)=\bar{P}\left(Q_{t}\left(e_{u}\right)\right)$ for all $t>0$ and $u$ from the cone $\mathcal{C}$ given by $(*)$. 
Since $\bar{L}_{\beta^{\prime}}(\xi, 1) \leq 1$, there exists a measure $v$ on $M(E)_{1}$ such that $\xi=v \circ \bar{U}_{\beta^{\prime}}$. Notice that every $f \in\left[\mathrm{b} \mathcal{E}\left(\overline{\mathcal{U}}_{\beta^{\prime}}\right)\right]$ has a finely continuous extension $\tilde{f}$ to $M(E)_{1}$. Let $\tilde{\mathcal{A}}$ be the linear space of bounded functions on $M(E)_{1}$ spanned by the set $\left\{\widetilde{e}_{u} \mid u \in \mathcal{S}\right\}$. Because $\widetilde{\mathcal{A}}$ is an algebra and $v\left(\widetilde{e}_{u}\right)=\bar{L}_{\beta^{\prime}}\left(\xi, e_{u}\right)=\bar{P}\left(\widetilde{e}_{u}\right)$ for every $u \in \mathcal{S}$, by the monotone class theorem we have

$$
v(F)=\bar{P}(F) \quad \text { for all } F \in \sigma(\widetilde{\mathcal{A}}) .
$$

By hypothesis $(*)$, if $u \in \mathcal{C}$ then there exists a sequence $\left(f_{n}\right)_{n} \subset[\mathcal{S}]$ converging uniformly to $V_{t} u$. As a consequence, for every $\mu \in M\left(\underset{\sim}{E)}\right.$ we have $\left|e_{f_{n}}(\mu)-e_{V_{t} u}(\mu)\right| \leq$ $\left\|f_{n}-V_{t} u\right\|_{\infty} \cdot l_{1}(\mu)$, hence $\left|\widetilde{e}_{f_{n}}-\widetilde{e}_{V_{t} u}\right| \leq\left\|f_{n}-V_{t} u\right\|_{\infty} \cdot \widetilde{l}_{1}$ on $M(E)_{1}$. It follows that $\left(\widetilde{e}_{f_{n}}\right)_{n}$ converges pointwise to $\widetilde{e}_{V_{t} u}$ on the set $\left[\widetilde{l}_{1}<\infty\right] \in \sigma(\widetilde{\mathcal{A}})$. From $v\left(\widetilde{l}_{1}\right)=\bar{L}_{\beta^{\prime}}\left(\xi, l_{1}\right)<\infty$ we deduce that $\tilde{l}_{1}<\infty, \nu$-a.e. Therefore, $1_{\left[\tilde{l}_{1}<\infty\right]} \cdot \widetilde{e}_{V_{t} u}$ is $\sigma(\widetilde{\mathcal{A}})$-measurable and by (4.3) we now get $v\left(\widetilde{e}_{V_{t} u}\right)=\bar{P}\left(\widetilde{e}_{V_{t} u}\right)$. Since $Q_{t}\left(e_{u}\right)=e_{V_{t} u}$, we conclude that

$$
\bar{L}_{\beta^{\prime}}\left(\xi, Q_{t}\left(e_{u}\right)\right)=v\left(\widetilde{e}_{V_{t} u}\right)=\bar{P}\left(Q_{t}\left(e_{u}\right)\right) .
$$

Notice that it follows from Corollary 4.3 that the weak Ray topology on $M(E)$ is natural for $\overline{\mathcal{U}}_{\beta^{\prime}}$. Consequently, the weak topology on $M(E)$ is also natural. Assertion (i) and the last assertion of (iii) follow now by (2.5) applied to $\overline{\mathcal{U}}_{\beta^{\prime}}$ instead of $\mathcal{U}$.

Step II. If we put $u_{o}:=R_{b^{\prime}}^{E_{1} \backslash E} 1$ then, since $B_{b^{\prime}}^{E_{1} \backslash E} 1=0$, we have $u_{o}=1_{E_{1} \backslash E}$, and therefore

$$
M(E)=\left[l_{u_{o}}=0\right] \in \mathcal{M}\left(E_{1}\right) .
$$

For every $\mu \in M\left(E_{1}\right)$ there exists a decreasing sequence $\left(v_{n}\right)_{n} \subset \mathrm{b} \mathcal{E}\left(\mathcal{U}_{b^{\prime}}\right)$ such that $v_{n} \geq u_{o}$ for every $n$ and $l_{u_{o}}(\mu)=\inf _{n} l_{v_{n}}(\mu)$. Therefore, we have $l_{u_{o}}=\inf \left\{F \in \mathcal{E}\left(\overline{\mathcal{U}}_{\beta^{\prime}}\right) \mid\right.$ $F \geq l_{u_{o}}$. According to [2], the function $l_{u_{o}}$ is strongly supermedian with respect to $\overline{\mathcal{U}}_{\beta^{\prime}}$ and so the set $M(E)=\left[l_{u_{o}}=0\right]$ is a finely open subset of $M\left(E_{1}\right)$ and

$$
\bar{R}_{\beta^{\prime}}^{M\left(E_{1}\right) \backslash M(E)} 1=1_{M\left(E_{1}\right) \backslash M(E)} .
$$

Because $U_{b^{\prime}}^{1}\left(u_{o}\right)=0$, it follows by Proposition 4.2 that $\bar{U}_{\beta^{\prime}}\left(l_{u_{o}}\right)=l_{U_{b^{\prime}}^{1}\left(u_{o}\right)}=0$. Consequently, $M\left(E_{1}\right)$ is embedded in $M(E)_{1}$. Since from Step I of the proof the resolvent $\overline{\mathcal{U}}$ on $M\left(E_{1}\right)$ is associated with a right process with state space $M\left(E_{1}\right)$, by (4.4) we can restrict this process to $M(E)$, obtaining the right process having $\left(Q_{t}\right)_{t \geq 0}$ as transition function.

Assertion (iv) follows from Proposition 4.7, relation (3.3) and since by Hunt's theorem on balayages we have $R_{\beta}^{G} f(x)=E^{x}\left(e^{-\beta T_{G}} f\left(X_{T_{G}}\right)\right)$.

Step III. Assume that $X$ is a Hunt process. From Theorem (47.10) in [17] we deduce that $X$ has càdlàg trajectories in any Ray topology. We consider such a Ray topology $\mathcal{T}_{\mathcal{R}}$ which is finer than the original topology and is generated by a Ray cone $\mathcal{R} \supset \mathcal{C}$. Let $\lambda \in M(E)$. By (4.2) there exists a function $v \in \mathcal{E}\left(\mathcal{U}_{b^{\prime}}\right) \cap L^{1}(E, \lambda)$ having $\mathcal{T}_{\mathcal{R}}$-compact level sets. Corollary 4.3 implies that $l_{v} \in \mathcal{E}\left(\overline{\mathcal{U}}_{\beta^{\prime}}\right)$ and it follows by [7] that $l_{v}$ has compact level sets 
in the weak Ray topology. According to (4.1), there exists an increasing sequence $\left(M_{n}\right)_{n}$ of weak Ray compact subsets of $M(E)$ such that

$$
\inf _{n} \bar{R}_{\beta^{\prime}}^{M(E) \backslash M_{n}} 1(\lambda)=0 .
$$

In order to prove that the $(X, \Phi)$-superprocess has càdlàg trajectories and it is quasi-leftcontinuous, we proceed as in the proof of Theorem 3.7.7 from [2].

We first show that the superprocess has $\bar{P}^{\lambda}$-a.s. left limits in $M(E)$. Let $\bar{E}$ be the Ray compactification of $E$ with respect to $\mathcal{R}$. Since by Corollary 4.3 the function $1-e_{u}$ is $\overline{\mathcal{U}}_{\beta^{\prime \prime}}-$ excessive (provided that $u \in \mathcal{R}$, for some $\beta^{\prime \prime}>\beta^{\prime}$ ), the process $t \mapsto e^{-\beta^{\prime \prime} t}\left(1-e_{u}\right)\left(X_{t}^{\Phi}\right)$ is a bounded right continuous supermartingale. We infer that this process has left limits $\bar{P}^{\lambda}$. a.s. (cf. [8]). Since the Ray cone $\mathcal{R}$ is separable with respect to the uniform norm, the process $\left(X_{t}^{\Phi}\right)_{t \geq 0}$ has $\bar{P}^{\lambda}$-a.s. left limits in $M(\bar{E})$. From $\bar{R}_{\beta^{\prime}}^{M(E) \backslash M_{n}} 1(\lambda)=\bar{E}^{\lambda}\left(e^{\left.-\beta^{\prime} T_{M(E) \backslash M_{n}}\right)}\right.$ we deduce by (4.5) that $\bar{P}^{\lambda}$-a.s. we have $\sup _{n} T_{M(E) \backslash M_{n}}=\infty$. Hence for every $\omega \in \Omega$ with $T_{M(E) \backslash M_{n}}(\omega)<\infty$ we have $X_{t}^{\Phi}(\omega) \in M_{n}$ provided that $t<T_{M(E) \backslash M_{n}}(\omega)$ and so $X_{t-}^{\Phi}(\omega) \in M_{n}$. Consequently, the process $\left(X_{t}^{\Phi}\right)_{t \geq 0}$ has left limits (in the weak Ray topology) in $M(E), \bar{P}^{\lambda}$-a.s. on $[0, \infty)$.

Let now $\left(T_{n}\right)_{n}$ be an increasing sequence of stopping times and $T=\lim _{n} T_{n}$. We show that $\lim _{n} X_{T_{n}}^{\Phi}=X_{T}^{\Phi}, \bar{P}^{\lambda}$-a.s. It follows from the above considerations that the limit $Z=\lim _{n} X_{T_{n}}^{\Phi}$ exists in $M(E), \bar{P}^{\lambda}$-a.s. It remains to prove that $Z=X_{T}^{\Phi}, \bar{P}^{\lambda}$-a.s. Let $u, v \in \mathcal{C}, G:=e_{u}$ and $F:=e_{v}$. If $\alpha>0$ and $n<m$ then

$$
\begin{aligned}
\bar{E}^{\lambda}\left(G\left(X_{T_{n}}^{\Phi}\right) \bar{U}_{\beta^{\prime}+\alpha} F\left(X_{T_{m}}^{\Phi}\right)\right) & =\bar{E}^{\lambda}\left(G\left(X_{T_{n}}^{\Phi}\right) \int_{0}^{\infty} e^{-\left(\beta^{\prime}+\alpha\right) t} e_{V_{t} v}\left(X_{T_{m}}^{\Phi}\right) d t\right) \\
& =\bar{E}^{\lambda}\left(G\left(X_{T_{n}}^{\Phi}\right) \int_{T_{m}}^{\infty} e^{-\left(\beta^{\prime}+\alpha\right)\left(t-T_{m}\right)} F\left(X_{t}^{\Phi}\right) d t\right) .
\end{aligned}
$$

Assuming that $V_{t}(\mathcal{C}) \subset \mathcal{C} \subset \mathcal{R}$ and letting $m \rightarrow \infty$, we obtain

$$
\begin{aligned}
\bar{E}^{\lambda}\left(G\left(X_{T_{n}}^{\Phi}\right) \bar{U}_{\beta^{\prime}+\alpha} F(Z)\right) & =\bar{E}^{\lambda}\left(G\left(X_{T_{n}}^{\Phi}\right) \int_{T}^{\infty} e^{-\left(\beta^{\prime}+\alpha\right)(t-T)} F\left(X_{t}^{\Phi}\right) d t\right) \\
& =\bar{E}^{\lambda}\left(G\left(X_{T_{n}}^{\Phi}\right) \bar{U}_{\beta^{\prime}+\alpha} F\left(X_{T}^{\Phi}\right)\right) .
\end{aligned}
$$

Letting now $n \rightarrow \infty$ we get

$$
\bar{E}^{\lambda}\left(G(Z) \alpha \bar{U}_{\beta^{\prime}+\alpha} F(Z)\right)=\bar{E}^{\lambda}\left(G(Z) \alpha \bar{U}_{\beta^{\prime}+\alpha} F\left(X_{T}^{\Phi}\right)\right) .
$$

Because $F \in\left[\mathrm{b} \mathcal{E}\left(\overline{\mathcal{U}}_{\beta^{\prime}}\right)\right]$, letting $\alpha \rightarrow \infty$, we get

$$
\bar{E}^{\lambda}(G(Z) F(Z))=\bar{E}^{\lambda}\left(G(Z) F\left(X_{T}^{\Phi}\right)\right) .
$$

The $\sigma$-algebra $\mathcal{M}(E)$ being generated by $\left\{e_{u} \mid u \in \mathcal{C}\right\}$, a monotone class argument implies that the above equality holds for all $F, G \in \mathrm{p} \mathcal{M}(E)$ and, as a consequence, 
$\bar{E}^{\lambda}(H(Z, Z))=\bar{E}^{\lambda}\left(H\left(Z, X_{T}^{\Phi}\right)\right)$ for all $H \in \mathrm{p}(\mathcal{M}(E) \times \mathcal{M}(E))$. Taking as $H$ the characteristic function of the diagonal of $M(E) \times M(E)$, we conclude that $\bar{P}^{\lambda}\left(\left[Z \neq X_{T}^{\Phi}\right]\right)=0$.

Remark. (i) Theorem 4.9 (iv) gives a relation between the hitting distributions of the finely open sets of the given spatial Markov process $X$ and the hitting distributions of the $(X, \Phi)$-superprocess. Notice that an analogous result for the excessive functions was stated in Corollary 4.3 namely the $\mathcal{U}_{b^{\prime}}$-excessive functions were identified (through the map $u \mapsto l_{u}$ ) with the "linear" $\overline{\mathcal{U}}_{\beta^{\prime}}$-excessive functions.

(ii) The additional assumption from Theorem 4.9(ii) is satisfied if condition (4.a) from Proposition 4.8 holds. One can check that in the proof of Theorem 4.9(ii) we use only the following weaker assumption: $V_{t}(\mathcal{C})$ is a subset of the closure of $[\mathcal{C}]$ in the supremum norm. This condition is satisfied if (4.b) holds.

(iii) The two integrability conditions imposed (in Section 1) on the kernel $N$ are precisely those considered in Theorem 3.2, Section 4.3.3 of [11] and are clearly satisfied if the assumption $N\left(u \vee u^{2}\right) \in \mathrm{bp} \mathcal{B}$ from [13] holds.

\section{Appendix}

Proof of Proposition 3.1. Let $F:=E \times(0, \infty)$. We shall consider on $(F, \mathcal{B}(F))$ the semigroup having the infinitesimal generator the "heat operator" $A-d / d t$. More precisely, if $h: E \times(0, \infty) \rightarrow \mathbb{R}$ is $\mathcal{B}(F)$-measurable, then we put

$$
\widetilde{P}_{t} h(x, s):= \begin{cases}P_{t} h(x, s-t) & \text { if } s>t \\ 0 & \text { if } s \leq t\end{cases}
$$

One can check that $\left(\widetilde{P}_{t}\right)_{t \geq 0}$ is a sub-Markovian semigroup of kernels on $(F, \mathcal{B}(F))$. Let $\left(\widetilde{V}_{\alpha}\right)_{\alpha>0}$ be its associated resolvent, so that

$$
\widetilde{V}_{\alpha} h(x, t)=\int_{0}^{t} e^{-\alpha s} P_{s} h(\cdot, t-s)(x) d s .
$$

Clearly, the initial kernel $\widetilde{V}$ of $\left(\widetilde{V}_{\alpha}\right)_{\alpha>0}$ is given by

$$
\widetilde{V} h(x, t)=\int_{0}^{t} P_{s} h(\cdot, t-s)(x) d s .
$$

We shall give two properties of $\left(\widetilde{V}_{\alpha}\right)_{\alpha>0}$.

(A.1) If $\beta>0$ and $f \in \mathrm{bp} \mathcal{B}$, then

$$
(I+\beta \tilde{V}) P^{\beta} f=P f \quad \text { on } F,
$$

where $P f(x, t):=P_{t} f(x)$ and $P^{\beta} f(x, t):=P_{t}^{\beta} f(x)$. 
Indeed, we have $(I+\beta \tilde{V}) P^{\beta} f(x, t)=\beta \int_{0}^{t} P_{s} P_{t-s}^{\beta} f(x) d s+P_{t}^{\beta} f(x)=e^{-\beta t} P_{t} f(x)+$ $e^{-\beta t} P_{t} f(x) \int_{0}^{t} \beta e^{\beta s} d s=P_{t} f(x)$.

(A.2) If $f, g \in \mathrm{p} \mathcal{B}(F)$ and $(I+\beta \tilde{V}) f=(I+\beta \tilde{V}) g<\infty$ then $f=g$.

The assertion follows directly from the resolvent equation.

Equation (1.1) is equivalent to

$$
v=P f+\widetilde{V} \Phi(\cdot, v)
$$

while (3.1) is equivalent to

$$
v=P^{\beta} f+\widetilde{V}_{\beta} \Phi_{\beta}(\cdot, v),
$$

where the function $v \in \mathrm{p} \mathcal{B}(F)$ is defined by $v(x, t):=v_{t}(x)$.

Since $\widetilde{V} v<\infty$, by (A.2) and (A.1) we have

$$
\begin{aligned}
v=P^{\beta} f+\widetilde{V}_{\beta} \Phi_{\beta}(\cdot, v) & \Leftrightarrow(I+\beta \tilde{V}) v=(I+\beta \tilde{V})\left(P^{\beta} f+\widetilde{V}_{\beta} \Phi_{\beta}(\cdot, v)\right) \\
& \Leftrightarrow v+\beta \widetilde{V} v=P f+\widetilde{V} \Phi_{\beta}(\cdot, v) \\
& \Leftrightarrow v=P f+\widetilde{V} \Phi(\cdot, v) .
\end{aligned}
$$

Hence (1.1) and 3.1) are equivalent.

Sketch of the proof of Proposition 3.2. As we mentioned in Introduction, this proof is due to P. J. Fitzsimmons (private communication).

Step I. Assume that $c \equiv 0$ and $\int_{0}^{1} s N(\cdot, d s) \in \mathrm{bp} \mathcal{B}$. The function $V_{t} f$ is constructed by the method of Picard iterations.

I.1. Let us fix $\lambda_{o}, t_{o}>0$ and define $\lambda_{1}:=\lambda_{o} e^{\beta t_{o}}$. Let $a$ be a real number with $a \geq$ $M+\|b\|_{\infty}$, where $\int_{0}^{\infty} s N(\cdot, d s) \leq M$. If we define the function $\varphi$ by

$$
\varphi(x, \lambda):=\Phi(x, \lambda)+a \lambda, \quad x \in E, \lambda \in \mathbb{R}_{+},
$$

we deduce that

$$
0 \leq \varphi(x, \lambda) \leq(a+\beta) \lambda \quad \text { for all } x \in E \text { and } \lambda \in\left[0, \lambda_{1}\right],
$$

and $\varphi$ is a Lipschitz function on $\left[0, \lambda_{1}\right]$ with Lipschitz constant $C>0$ (uniformly in $x \in E)$.

For $f \in \mathrm{bp} \mathcal{B}, f \leq \lambda_{o}$, and $x \in E$ we define $V_{t}^{0} f(x)=0$ and, for $k \geq 0$,

$$
V_{t}^{k+1} f(x)=P_{t}^{a} f(x)+\int_{0}^{t} P_{s}^{a}\left(x, \varphi\left(\cdot, V_{t-s}^{k} f\right)\right) d s, \quad 0 \leq t \leq t_{o},
$$

where $P_{t}^{a}:=e^{-a t} P_{t}$. One can prove that

$$
0 \leq V_{t}^{k} f(x) \leq e^{\beta t}\|f\|_{\infty}
$$


for all $x \in E$, and

$$
\left\|V_{t}^{k+1} f-V_{t}^{k} f\right\|_{\infty} \leq \frac{(C t)^{k}}{k !}\|f\|_{\infty} .
$$

The last inequality implies that the limit

$$
V_{t} f(x)=\lim _{k} V_{t}^{k} f(x)
$$

exists uniformly in $(x, t) \in E \times\left[0, t_{o}\right]$. In particular, the function $v_{t}(x):=V_{t} f(x)$, $x \in E$, is a solution of the equation

$$
v_{t}(x)=P_{t}^{a} f(x)+\int_{0}^{t} P_{s}^{a}\left(x, \varphi\left(\cdot, v_{t-s}\right)\right) d s, \quad 0 \leq t \leq t_{o} .
$$

The uniqueness for equation (1.1) follows by Gronwall's lemma while the semigroup property (assertion (iv)) is a consequence of this. Varying $\lambda_{o}$ and $t_{o}$, we obtain the existence and uniqueness for equation (A.3) for every $f \in \mathrm{bp} \mathcal{B}$. By Proposition 3.1 we now deduce that assertions (i) and (ii) hold. Assertion (iii) follows since the right continuity of the map $t \mapsto P_{t} f(x)$ implies the same property for $t \mapsto V_{t}^{k} f(x)$ for every $k \geq 0$.

I.2. The negative definiteness. We show that if the map $V: \mathrm{bp} \mathcal{B} \rightarrow \mathrm{bp} \mathcal{B}$ is such that $f \mapsto V f(x)$ is negative definite for every $x \in E$, then so also is $f \mapsto \varphi(x, V f(x))$. Indeed, the assertion follows from (2.6) because

$$
\varphi(x, \lambda)=\left(a-b(x)-\int_{0}^{\infty} s N(x, d s)\right) \lambda+\int_{0}^{\infty}\left(1-e^{-\lambda s}\right) N(x, d s)
$$

and $a \geq b+\int_{0}^{\infty} s N(\cdot, d s)$. Notice that the map $f \mapsto P_{t}^{a} V f(x)$ is also negative definite. We conclude by induction that $f \mapsto P_{t}^{a} V^{k} f(x)$ is negative definite for every $k$, and therefore also $f \mapsto V_{t} f(x)$ as a limit of negative definite functions.

Step II (The general case for $c$ and $N)$. For $\eta \in(0,1)$ define

$$
\Phi^{\eta}(x, \lambda)=-b(x) \lambda+\int_{0}^{\infty}\left(1-e^{-\lambda s}-\lambda s\right) N^{\eta}(x, d s)
$$

where

$$
N^{\eta}(x, d s):=\frac{2 c(x)}{\eta^{2}} \varepsilon_{\eta}(d s)+1_{[s>\eta]} N(x, d s) .
$$

Then $\int_{0}^{1} s N^{\eta}(\cdot, d s) \in \mathrm{bp} \mathcal{B}, \Phi^{\eta}$ is also a Lipschitz function on $\left[0, \lambda_{1}\right]$ and by the hypothesis on $N$,

$$
\lim _{\eta \rightarrow 0} \sup _{\lambda \leq \lambda_{1}} \frac{1}{\lambda^{2}}\left\|\Phi^{\eta}(\cdot, \lambda)-\Phi(\cdot, \lambda)\right\|_{\infty}=0 .
$$

If $V_{t}^{\eta} f$ denotes the solution of equation 1.1 with $N^{\eta}$ instead of $N$, then by Gronwall's lemma $V_{t}^{\eta} f$ converges uniformly in $x$ and $t \leq t_{o}$ to the solution $V_{t} f$ of $[1.1]$ as $\eta \rightarrow 0$.

Assertion (vi) holds since a consequence of (iv) is the fact that the map $f \mapsto V_{t} f(x)$ is increasing and by the uniqueness of the solution of 1.1 . 
Proof of Proposition 3.3. As in the proof of (2.7) from [13], the existence and uniqueness of the solution of equation (3.2) follows from Proposition 3.2 applied for $\Phi(x, \lambda)=$ $-b(x) \lambda$. Equation (3.3) follows by uniqueness. The inequalities $e^{-\beta^{\prime} t} P_{t} \leq P_{t}^{b} \leq e^{\beta t} P_{t}$, $P_{t}^{b_{2}} \leq P_{t}^{b_{1}}$ if $b_{1} \leq b_{2}$, and the first assertion of (iii) are consequences of (3.3). In particular, we get $P_{t}^{\bar{b}} 1>0$.

Assertion (ii) follows since by Proposition 3.2 (iii) the resolvent $\mathcal{U}_{\beta}$ is exactly subordinate to $\mathcal{U}$, and by Section 5.1 in [2]. To prove that $\left(P_{t}^{b_{2}-b_{1}}\right)^{b_{1}}=P_{t}^{b_{2}}$, let $S_{t}:=\left(P_{t}^{b_{2}-b_{1}}\right)^{b_{1}}$. It is sufficient to show that for all $t \geq 0$ we have

$$
S_{t} f=P_{t} f-\int_{0}^{t} P_{s}\left(b_{2} S_{t-s} f\right) d s
$$

Since $S_{t}=P_{t}^{b_{2}-b_{1}}-\int_{0}^{t} P_{s}^{b_{2}-b_{1}}\left(b_{1} S_{t-s}\right) d s$ and $P_{s}^{b_{2}-b_{1}}=P_{s}-\int_{0}^{s} P_{u}\left(\left(b_{2}-b_{1}\right) P_{s-u}^{b_{2}-b_{1}}\right) d u$, we get

$$
P_{t}^{b_{2}-b_{1}}-S_{t}=\int_{0}^{t} d s\left(P_{s}-\int_{0}^{s} P_{u}\left(\left(b_{2}-b_{1}\right) P_{s-u}^{b_{2}-b_{1}}\right) d u\right)\left(b_{1} S_{t-s}\right)
$$

thus

$$
\begin{gathered}
S_{t}-P_{t}^{b_{2}-b_{1}}+\int_{0}^{t} P_{s}\left(b_{1} S_{t-s}\right) d s=\int_{0}^{t} d s \int_{0}^{s} P_{u}\left(\left(b_{2}-b_{1}\right) P_{s-u}^{b_{2}-b_{1}}\left(b_{1} S_{t-s}\right)\right) d u \\
=\int_{0}^{t} d u\left[P_{u}\left(\left(b_{2}-b_{1}\right) \int_{0}^{t-u} P_{s}^{b_{2}-b_{1}}\left(b_{1} S_{(t-u)-s}\right) d s\right)\right] \\
=\int_{0}^{t} P_{u}\left(\left(b_{2}-b_{1}\right)\left(P_{t-u}^{b_{2}-b_{1}}-S_{t-u}\right)\right) d u, \\
S_{t}=P_{t}^{b_{2}-b_{1}}+\int_{0}^{t} P_{u}\left(\left(b_{2}-b_{1}\right) P_{t-u}^{b_{2}-b_{1}}\right) d u-\int_{0}^{t} P_{u}\left(b_{2} S_{t-u}\right) d u=P_{t}-\int_{0}^{t} P_{s}\left(b_{2} S_{t-s}\right) d s .
\end{gathered}
$$

Proof of Proposition 4.1. By Proposition 3.2(v)\&(vi) and (2.6), the map $f \mapsto e_{V_{t} f}(\mu)$ is positive definite on bp $\mathcal{B}$ for every $\mu \in M(E)$, and if $\left(f_{n}\right)_{n} \subset$ bp $\mathcal{B}$ is pointwise decreasing to zero, then $e_{V_{t} f_{n}} \nearrow e_{V_{t} 0}$. By Corollary 2.6 there exists a unique finite measure $Q_{t, \mu}$ on $(M(E), \mathcal{M}(E))$ such that $Q_{t, \mu}\left(e_{f}\right)=e_{V_{t} f}(\mu)$ for every $f \in \mathrm{bp} \mathcal{B}$. Put

$$
Q_{t} F(\mu):=Q_{t, \mu}(F), \quad F \in \operatorname{bp} \mathcal{M}(E) .
$$

Since $Q_{t}\left(e_{f}\right) \in \operatorname{bp} \mathcal{M}(E)$ for every $f \in \operatorname{bp} \mathcal{B}$ and the set $\left\{\sum_{i=1}^{n} a_{i} e_{f_{i}} \mid n \in \mathbb{N}^{*}, f_{i} \in\right.$ $\left.\mathrm{bp} \mathcal{B}, a_{i} \in \mathbb{R}, i \leq n\right\}$ is an algebra of bounded $\mathcal{M}(E)$-measurable functions generating $\mathcal{M}(E)$, we conclude that $Q_{t} F \in \operatorname{bp} \mathcal{M}(E)$ for every $F \in \operatorname{bp} \mathcal{M}(E)$, hence $Q_{t}$ is a kernel on $(M(E), \mathcal{M}(E))$.

Proof of Proposition 4.2. We define the function $\varphi_{t}: \mathbb{R}_{+} \rightarrow$ bp $\mathcal{B}$ by $\varphi_{t}(\lambda):=V_{t} \lambda f$. Since the map $f \mapsto V_{t} f(x)$ is negative definite, it is increasing, and using Corollary 2.6 . 
it is concave. The function $\lambda \mapsto \varphi_{t}(\lambda) / \lambda$ is therefore decreasing and by Proposition 3.2.ii) we have

$$
\frac{\varphi_{t}(\lambda)}{\lambda} \leq e^{\beta t}\|f\|_{\infty}
$$

Therefore, the function $\varphi_{t}^{\prime}(0):=\sup _{\lambda>0} \varphi_{t}(\lambda) / \lambda$ belongs to bp $\mathcal{B}$ and, in addition, for every $\mu \in M(E)$ we have

$$
l_{\varphi_{t}^{\prime}(0)}(\mu)=\lim _{\lambda \searrow 0} \frac{1-e_{\varphi_{t}(\lambda)}(\mu)}{\lambda} .
$$

On the other hand, $Q_{t}\left(e_{\lambda f}\right)=e_{\varphi_{t}(\lambda)}$, and therefore $l_{\varphi_{t}^{\prime}(0)}(\mu)=-\left(Q_{t}\left(e_{\lambda f}\right)(\mu)\right)_{\lambda=0}^{\prime}=$ $Q_{t} l_{f}(\mu)$. Hence it is sufficient to show that $\varphi_{t}^{\prime}(0)$ satisfies equation 3.2. We have

$$
\begin{aligned}
\frac{\varphi_{t}(\lambda)}{\lambda}= & P_{t} f-\int_{0}^{t} P_{t-s}\left(b \frac{\varphi_{s}(\lambda)}{\lambda}\right) d s \\
& -\int_{0}^{t} P_{t-s}\left(c \frac{\varphi_{s}^{2}(\lambda)}{\lambda}+\int_{0}^{\infty} \frac{1}{\lambda}\left[1-e^{\varphi_{s}(\lambda) u}-\varphi_{s}(\lambda) u\right] N(\cdot, d u)\right) d s .
\end{aligned}
$$

Letting $\lambda \rightarrow 0$ we find that $\varphi_{t}^{\prime}(0)$ satisfies 3.2 since the last two terms converge to zero by (A.4) and because $\lim _{\lambda \searrow 0} \varphi_{S}(\lambda)=0$.

Notes added in proof. 1. In Zenghu Li's monograph [16], devoted to measure-valued branching processes, it is shown (by a counterexample in Section 5.4) that the hypothesis on $X$ to be a Hunt process in Theorem 4.9(ii) is necessary in order to deduce that the $(X, \Phi)$-superprocess is also a Hunt process.

2. In the proof of Theorem 4.9, a main step in obtaining the càdlàg property of the trajectories of the measure-valued $(X, \Phi)$-superprocess was the existence of a nest of weak compact sets on the space of measures, produced by a special excessive function having compact level sets. It turns out that this is an efficient way to obtain the path regularity of a Markov process in other infinitedimensional situations too; a presentation of this method and its applications in relevant examples are given in the survey article [6].

3. In [5] a Markov process is constructed which is a combination of an $(X, \Phi)$-superprocess and a discrete branching type process, on the space of finite configurations of positive finite measures.

Acknowledgments. Support from the Romanian Ministry of Education, Research, and Innovation (PN II Program, CNCSIS code 1186/2008) is gratefully acknowledged.

\section{References}

[1] Berg, C., Christensen, J. P. R., Ressel, P.: Harmonic Analysis on Semigroups. Springer (1984) Zbl 0619.43001 MR 0747302

[2] Beznea, L., Boboc, N.: Potential Theory and Right Processes. Math. Appl. 572, Kluwer (2004) Zbl 1091.31010 MR 2153655

[3] Beznea, L., Boboc, N., Röckner, M.: Markov processes associated with $L^{p}$-resolvents and applications to stochastic differential equations on Hilbert space. J. Evol. Equations 6, 745772 (2006) Zbl 1114.60060 MR 2267706 
[4] Beznea, L., Cornea, A., Röckner, M.: Compact excessive functions and Markov processes: a general case and applications. In: Proc. RIMS Workshop on Stochastic Analysis and Applications RIMS, Kokyuroku Bessatsu B6, Kyoto, 31-37 (2008)

[5] Beznea, L., Oprina, A.-G.: Nonlinear PDEs and measure-valued branching type processes. J. Math. Anal. Appl. (2011), doi:10.1016/j.jmaa.2010.10.034

[6] Beznea, L., Röckner, M.: Applications of compact superharmonic functions: path regularity and tightness of capacities. Complex Anal. Oper. Theory (2011), doi:10.1007/s11785-0100084-3

[7] Bogachev, V. I.: Measure Theory (Vol. I, II). Springer (2007) Zbl 1120.28001 MR 2267655

[8] Dellacherie, C., Meyer, P. A.: Probabilités et potentiel (ch. I-IV, IX-XI, XII-XVI). Hermann, Paris $(1975,1983,1987)$ Zbl 0323.60039(I-IV) Zbl 0526.60001(IX-XI) Zbl 0624.60084(XII-XVI) MR 0488194(I-IV) MR 0727641(IX-XI) MR 0960549 (XII-XVI)

[9] Dynkin, E. B.: Three classes of infinite-dimensional diffusions. J. Funct. Anal. 86, 75-110 (1989) Zbl 0683.60040 MR 1013934

[10] Dynkin, E. B.: On the regularity of superprocesses. Probab. Theory Related Fields 95, 263281 (1993) Zbl 0791.60075 MR 1214090

[11] Dynkin, E. B.: Diffusions, Superdiffusions and Partial Differential Equations. Amer. Math. Soc. Colloq. Publ. 50, Amer. Math. Soc. (2002) Zbl 0999.60003 MR 1883198

[12] El Karoui, N., Roelly, S.: Propriétés de martingales, explosion et représentation de LévyKhinchine d'une classe de processus de branchement à valeurs mesures. Stoch. Process. Appl. 38, 239-266 (1991) Zbl 0743.60081 MR 1119983

[13] Fitzsimmons, P. J.: Construction and regularity of measure-valued Markov branching processes. Israel J. Math. 64, 337-361 (1988) Zbl 0673.60089 MR 1119935

[14] Iscoe, I.: A weighted occupation time for a class of measure-valued branching processes. Probab. Theory Related Fields 71, 85-116 (1986) Zbl 0555.60034 MR 0814663

[15] Leduc, G.: The complete characterization of a general class of superprocesses. Probab. Theory Related Fields 116, 317-358 (2000) Zbl 0955.60079 MR 1749278

[16] Li, Z. H.: Measure-Valued Branching Markov Processes. Probab. Appl., Springer (2011) Zbl pre05819419

[17] Sharpe, M.: General Theory of Markov Processes. Pure Appl. Math. 133, Academic Press (1988) Zbl 0649.60079 MR 0958914

[18] Steffens, J.: Excessive measures and the existence of right semigroups and processes. Trans. Amer. Math. Soc. 311, 267-290 (1989) Zbl 0658.60108 MR 0929664 